\title{
EXTERIOR BOUNDARY VALUE PROBLEMS FOR ELLIPTIC EQUATIONS
}

\author{
J. SARANEN and K. J. WITSCH
}

\section{Introduction}

In some exterior domain $\Omega \subset \mathbf{R}^{n}, n \geqq 2$, having a smooth boundary $\Gamma_{0}:=\partial \Omega$, we consider a strongly elliptic operator

$$
A:=\sum_{0 \leqq|\alpha|,|\beta| \leqq m}(-1)^{|\alpha|} \partial^{\alpha}\left(a_{\alpha \beta} \partial^{\beta}\right)
$$

of order $2 m, m>0$. Our aim is to prove Fredholm theorems with explicitly stated solvability conditions for the problem

$$
\begin{aligned}
& A u=f, \\
& \left.B_{j} u\right|_{\Gamma_{0}}=g_{j}, \quad j=0, \ldots, m-1,
\end{aligned}
$$

where the boundary operators

$$
B_{j} u:=\sum_{|v| \leqq m_{j}} b_{j v} \partial^{v} u
$$

of order $m_{j} \leqq 2 m-1$ are supposed to cover the operator $A$ and to be a normal system on the boundary $\Gamma_{0}$. The coefficients are assumed to be smooth on the closed domain $\bar{\Omega}=\Omega \cup \Gamma_{0}$. In addition to equations (1.2), we shall later impose an additional condition for the behaviour of the solution at infinity. This is necessary to guarantee that the boundary value problem is of Fredholm type. The conditions of solvability will be stated explicitly by means of an appropriate adjoint boundary value problem.

Our results will cover e.g. potential equations as well as problems of the radiation type.

In the case of bounded domains the solvability properties of the regular problem

The first author has been supported by der Deutsche Akademische Austauschdienst (DAAD), by Jenny and Antti Wihuri Foundation as well as by Alexander von Humboldt Foundation. The work was carried out during his visits at the University of Essen and at the Technical University of Darmstadt 1981-82. 
(1.2) in the Sobolev space $H^{2 m}(\Omega)$ for the data

$$
\left(f ; g_{0}, \ldots, g_{m-1}\right) \in L^{2}(\Omega) \times \prod_{j=0}^{m-1} H^{2 m-m_{j}-1 / 2}\left(\Gamma_{0}\right)
$$

are well known. In particular, the associated operator $u \rightarrow\left(A u ; B_{0} u, \ldots, B_{m-1} u\right)$ is an indexed operator and the conditions of the solvability of (1.2) can be described by an adjoint boundary value problem, Lions and Magenes [21], Theorem 5.3, p. 164.

No corresponding general results are known for exterior domains. On the other hand, there are numerous papers dealing with special problems of type (1.2) in exterior domains. To obtain a reasonable theory, the behaviour of the solutions at infinity has to be taken into account. We mention the main types of problems which have interest from a purely mathematical point of view as well as due to their applications in mathematical physics.

An important class of earlier papers considers problems where a "radiation condition" must be satisfied. The simplest example is the reduced wave equation where $A=\Delta+k^{2}, k>0$. This operator and its second order generalizations have been studied by Eŭdus [7], Jäger [13], Saito [30], Wilcox [42] and Witsch [43] among others. For earlier references, see the literature in these articles. These papers, apart from [42] and [43], deal with the Dirichlet boundary condition where the corresponding boundary value problem is uniquely solvable.

In [43] an oblique type boundary condition is considered. A Fredholm alternative is proved with explicitly stated solvability conditions.

Generalizations of the theory of the reduced wave equation, also called Helmholtz's equation, to higher order equations were given by Finoženok [9], Grušin [10], Vaŭnberg [35], [36] and Vogelsang [38], [39]. Apart from [36] only the whole space problem or the exterior Dirichlet problem is discussed. In [36], Vaĭnberg considers general regular boundary value problems and proves a Fredholm type result. However, the orthogonality conditions are not described.

The potential equation has also been solved in the whole space or in exterior domains, Courant and Hilbert [4], Kudrjavcev [16], Meyers and Serrin [22], Neittaanmäki [23] (fourth order), Saranen [33], Witsch [43]. For a comprehensive treatment including nonlinear problems with the Dirichlet boundary condition we refer to Edmunds and Evans [6]. In dealing with problems of this type, one uses function spaces which, roughly speaking, require that the functions fall to zero sufficiently rapidly at infinity. The results concerning the potential equation (Poisson equation) can be extended to other static problems, as we shall see in Section 4 of the present paper.

Whole space and exterior problems for polyharmonic equations and for more general related equations were considered by Paneyah [24], Saranen [32], Vekua [37] and Witsch [45]. They have defined the solution by a suitable decomposition method which reduces the problem to a system of lower order equations. In the exterior 
case the boundary conditions were of the Dirichlet- or Riquier-type, which in the polyharmonic case $A=\Delta^{m}$ prescribes $u, \Delta u, \ldots, \Delta^{m-1} u$ at the boundary (cf. [37], p. 347). The plate equation, which represents a fourth order equation and describes real physical phenomena, partly falls into this group. Related exterior problems have been studied by Leis [18], [19], Neittaanmäki [23], Polis [27], Saranen [31], Wickel [40], [41] and Witsch [44].

Here we give a unified approach to the general exterior boundary value problem (1.2) under the assumption that the corresponding Dirichlet problem can be solved for an exterior subdomain of $\Omega$. We use the alternating method, which goes back to Schwarz and was employed by Leis [17] and Witsch [43] in the case of exterior problems. It is worth observing that in contrast to these works no unique continuation property is needed in our modification of the alternating method. The importance of this lies in the fact that the unique continuation is not valid for all elliptic equations; for counter-examples see Plis [25], [26] and for the cases where this property has been verified see e.g. Calderón [3] and Protter [28].

The solution is built up from solutions of an exterior Dirichlet problem and a boundary value problem in a bounded domain, imposing the boundary conditions (1.2) on its boundary component $\Gamma_{0}$. The fact that the unique continuation property can be avoided is essentially due to an effective use of Fredholm inverses of the linear operators describing the auxiliary boundary problems.

According to our key result, Theorem 3.6, problem (1.2) has a finite index which is exactly the sum of the indices of the auxiliary problems mentioned above. Furthermore, we are able to give the orthogonality conditions for the solvability by means of an adjoint exterior boundary value problem.

In the remaining sections we shall apply Theorem 3.6 to various types of problems. In Section 4, potential type problems are discussed. These are the problems of the form (1.2) where the coefficients of the elliptic operator $A$, not belonging to the principal part, fall to zero sufficiently rapidly at infinity.

In Section 5 we achieve a Fredholm theorem for general elliptic radiation problems. Thereby we apply the results of Vogelsang [38], [39], derived for exterior Dirichlet problems. Thus, our Fredholm theorem for the radiation problems in this section is achieved essentially for the class of operators considered by Vogelsang.

For $x \rightarrow \infty$, the operators discussed in Section 5 have to tend to a limit operator $A_{\infty}(\partial)$ with constant coefficients. A crucial assumption on this limit operator is that the zeros of $A_{\infty}(\xi)$ are simple. This condition is violated in the case of products of Helmholtz operators, which we treat in the last section. 


\section{Preliminaries}

2.1. Notation. If $A$ is a subset of the Euclidean space $\mathbf{R}^{n}$, then $\bar{A}$ and $\partial A$ denote the closure and the boundary of $A$ in the topology of $\mathbf{R}^{n}$. If for two subsets $A$ and $B$ holds $\bar{A} \subset B$ and if $\bar{A}$ is compact, we write $A \subset \subset B$.

We use the standard Sobolev spaces $H^{s}(\Omega), s \geqq 0$ for functions in $\Omega$ and the boundary spaces $H^{s}(\Gamma), s \in \mathbf{R}$. For the definition of these spaces, see Lions and Magenes [21], p. 34, p. 40. Thus we have $L^{2}(\Omega)=H^{0}(\Omega)$ and $L^{2}(\Gamma)=H^{0}(\Gamma)$. In the spaces $H^{s}(\Omega)$ and $H^{s}(\Gamma)$ we use the norms $\|\cdot\|_{s, \Omega}$ and $\|\cdot\|_{s, \Gamma}$. When appropriate, the norm of the space $X$ is also denoted by $\|\cdot\|(X)$. The spaces $L^{2}(\Omega)$ and $L^{2}(\Gamma)$ are endowed with the usual $L^{2}$ inner products $(u \mid v)_{0, \Omega}$ and $\langle u \mid v\rangle_{0, \Gamma}$, respectively. The last notation is also employed for the sesquilinear duality pairing for the pair $H^{s}(\Gamma), H^{-s}(\Gamma)=\left(H^{s}(\Gamma)\right)$,' extending the $L^{2}(\Gamma)$ inner product.

2.2. Regular boundary value problems. We recall the contents of the essential notations in connection with regular boundary value problems. This notion is usually employed for boundary value problems in bounded domains. Here the same term also is used for exterior problems when the corresponding conditions without any "boundary condition at infinity" are satisfied.

Let

$$
A_{2 m}(x, \xi)=\sum_{|\alpha|=|\beta|=m} a_{\alpha \beta}(x) \xi^{\alpha+\beta}, \quad x \in \bar{\Omega}, \quad \xi \in \mathbf{R}^{n}
$$

be the characteristic form of the differential operator

$$
A=\sum_{0 \leqq|\alpha|,|\beta| \leqq m}(-1)^{|\alpha|} \partial^{\alpha}\left(a_{\alpha \beta} \partial^{\beta}\right)
$$

We assume that the coefficients $a_{\alpha \beta}$ are smooth in $\bar{\Omega}, a_{\alpha \beta} \in C^{\infty}(\bar{\Omega})$. The operator $A$ is assumed to be properly as well as strongly elliptic in $\bar{\Omega}$. The proper ellipticity of $A$ means that for any fixed $x \in \bar{\Omega}$ and linear independent vectors $\xi, \eta \in \mathbf{R}^{n}$ the polynomial $A_{2 m}(x, \xi+\tau \eta)$ in $\tau$ has exactly $m$ roots with positive and $m$ roots with negative imaginary parts. By the strong ellipticity in $\bar{\Omega}$ we assume that for any $R>0$

$$
\operatorname{Re} A_{2 m}(x, \xi) \geqq a_{0}(R)|\xi|^{2 m}, \quad(x, \xi) \in \bar{\Omega}(R) \times \mathbf{R}^{n},
$$

where $a_{0}(R)>0$ and $\bar{\Omega}(R):=\{x \in \bar{\Omega}:|x|<R\}$ (cf. [21], p. 110-111).

Furthermore, we suppose that there are given boundary operators $B_{j}$, $j=0, \ldots, m-1$ of order $m_{j} \leqq 2 m-1$ such that

$$
B_{j}=\sum_{|v| \leqq m_{j}} b_{j v}(x) \partial^{v}
$$

and that the coefficients $b_{j v}$ are smooth, $b_{j v} \in C^{\infty}\left(\Gamma_{0}\right)$.

For the following definitions see [21] p. 112-114. 
Definition 2.1. A system $\left\{D_{j}\right\}_{j=0}^{k}$ of boundary operators

is normal on $\Gamma_{0}$ if

$$
D_{j}:=\sum_{|v| \leqq k_{j}} d_{j v}(x) \partial^{v}
$$

(i) $k_{i} \neq k_{j}$ for $i \neq j$,

(ii) $\sum_{|v|=k_{j}} d_{j v}(x) \xi^{v} \neq 0$ for all $\xi \neq 0$ such that $\xi$ is a normal vector of $\Gamma_{0}$ at the point $x \in \Gamma_{0}$.

The system is a Dirichlet system of order $k$ if it is normal on $\Gamma_{0}$ and if the orders $k_{j}$ of $D_{j}$ form a permutation of the numbers $0,1, \ldots, k$.

Definition 2.2. The system $\left\{B_{j}\right\}_{j=0}^{m-1}$ covers the operator $A$ on $\Gamma_{0}$ if for all $x \in \Gamma_{\mathbf{0}}$ and $\xi \in \mathbf{R}^{n}, \xi \neq 0$, such that $\xi$ is tangential to $\Gamma_{\mathbf{0}}$ at $x$, and for all $\eta \in \mathbf{R}^{n}, \eta \neq 0$, such that $\eta$ is normal to $\Gamma_{0}$ at $x$, the complex polynomials of $\tau$, $\sum_{|v|=m_{j}} b_{j v}(x)(\xi+\tau \eta)^{v}, \quad j=0, \ldots, m-1$ are linearly independent modulo the polynomial $\prod_{i=1}^{m}\left(\tau-\tau_{i}^{+}(x, \xi, \eta)\right)$, where $\tau_{i}^{+}(x, \xi, \eta)$ are the roots of the polynomial $A_{2 m}(x, \xi+\tau \eta)$ in $\tau$ with positive imaginary part.

Finally, we recall the definition of a regular boundary value problem

$$
\begin{aligned}
& A u=f, \quad \text { in } \Omega, \\
& B_{j} u_{\Gamma_{0}}=g_{j}, \quad j=0, \ldots, m-1 .
\end{aligned}
$$

Let us first remark that regular boundary value problems (2.5) have been studied completely in bounded domains. The term "regular" does not require that the operator $A$ be strongly elliptic as supposed by us for exterior domains. For the regularity of (2.5) it is enough that the operator $A$ is properly elliptic. We shall point out where our stronger assumption comes into use.

Definition 2.3. Let $A$ be a properly elliptic operator of order $2 m$ in $\Omega$ with smooth coefficients in $\bar{\Omega}$ and let $\left\{B_{j}\right\}_{j=0}^{m-1}$ be a system of boundary operators $B_{j}$ with smooth coefficients in $\Gamma_{0}$ and of order $m_{j} \leqq 2 m-1$. Then the boundary value problem (2.5) is regular if $\left\{B_{j}\right\}_{j=0}^{m-1}$ is a normal system covering the operator $A$ on $\Gamma_{0}$.

For shortness we also say that the problem $\left(A ;\left\{B_{j}\right\}_{j=0}^{m-1}\right)$ is regular.

2.3. Solvability in bounded domains. In treating the exterior boundary value problem we shall make use of the solvability properties of regular problems in smooth bounded domains.

The exact statement of problem (2.5) in the space $H^{2 m}(\Omega)$ is given as follows. With the problem $\left(A,\left\{B_{j}\right\}_{j=0}^{m-1}\right)$ we associate an operator $\mathscr{P}: H^{2 m}(\Omega) \rightarrow L^{2}(\Omega) \times$ $\prod_{j=0}^{m-1} H^{2 m-m_{j}-1 / 2}\left(\Gamma_{0}\right)=: L^{2}(\Omega) \times X_{0}$ such that

$$
\mathscr{P} u:=\left(A u ; B_{0} u, \ldots, B_{m-1} u\right)=:(A u ; B u),
$$

where $B_{j} u \in H^{2 m-m_{j}-1 / 2}\left(\Gamma_{0}\right)$ is defined as a trace. Now, given the data $(f: g):=$ $\left(f ; g_{0}, \ldots, g_{m-1}\right) \in L^{2}(\Omega) \times X_{0}$, the function $u \in H^{2 m}(\Omega)$ is a solution of (2.5) if and only if we have $\mathscr{P} u=(f ; g)$. 
The essential tools in describing the solvability of problem (2.5) are Green's formula and an appropriate adjoint problem. Let $A^{*}$ be the formal adjoint of $A$

$$
A^{*}=\sum_{0 \leqq|\alpha|,|\beta| \leqq m}(-1)^{|\alpha|} \partial^{\alpha}\left(\bar{a}_{\beta \alpha} \partial^{\beta}\right)
$$

and let $C=\left\{C_{j}\right\}_{j=0}^{m-1}$ be a system of boundary operators $C_{j}$ with smooth coefficients and of order $\mu_{j} \leqq 2 m-1$ such that $\left\{C_{j}\right\}_{j=0}^{m-1}$ is adjoint to $\left\{B_{j}\right\}_{j=0}^{m-1}$ in the sense of [21], p. 121. This means that there exist two families $S=\left\{S_{j}\right\}_{j=0}^{m-1}$ and $T=\left\{T_{j}\right\}_{j=0}^{m-1}$ of boundary operators $S_{j}$ and $T_{j}$ with smooth coefficients and of order $2 m-1-\mu_{j}$ and $2 m-1-m_{j}$, respectively, such that the systems $\{B ; S\}$ and $\{C ; T\}$ are Dirichlet systems of order $2 m$, and that we have Green's formula

$$
(A u \mid v)_{0, \Omega}-\left(u \mid A^{*} v\right)_{0, \Omega}=\sum_{j=0}^{m-1}\left(\left\langle B_{j} u \mid T_{j} v\right\rangle_{0, \Gamma_{0}}-\left\langle S_{j} u \mid C_{j} v\right\rangle_{0, \Gamma_{0}}\right)
$$

for all $u, v \in H^{2 m}(\Omega)$, cf. [21], p. 114-115 with different notation.

The adjoint problem

$$
\begin{aligned}
& A^{*} u=f, \\
& \left.C_{j} u\right|_{\Gamma_{0}}=g_{j}, \quad j=0, \ldots, m-1,
\end{aligned}
$$

is given by the operator $\mathscr{P}^{*}: H^{2 m}(\Omega) \rightarrow L^{2}(\Omega) \times X_{0}$ such that

$$
\mathscr{P}^{*} u=\left(A^{*} u ; C_{0} u, \ldots, C_{m-1} u\right) .
$$

The adjoint boundary value problem is not uniquely defined. However, any adjoint problem makes it possible to describe the solvability conditions of boundary value problem (2.5). If, in particular, $B_{j} u=\gamma_{j} u=\left.(\partial / \partial n)^{j} u\right|_{\Gamma_{0}}$, then $C_{j}$ can be chosen as $C_{j}=\gamma_{j}$. We recall that the linear operator $T: X \rightarrow Y$, with normed spaces $X$ and $Y$, is an indexed operator if its kernel $N(T)$ is finite dimensional and if its range $R(T)$ is closed and has a finite codimension. The index $\varkappa(T)$ is given by

$$
\varkappa(T)=\operatorname{dim} N(T)-\operatorname{codim} R(T) .
$$

The solvability of regular problems in the space $H^{2 m}(\Omega)$ is settled by the following result ([21] p. 164).

Theorem 2.4. Let $\mathscr{P}=\left(A ; B_{0}, \ldots, B_{m-1}\right): H^{2 m}(\Omega) \rightarrow L^{2}(\Omega) \times X_{0}$ describe a regular boundary problem in the smooth bounded domain $\Omega$ and let $\mathscr{P}^{*}=\left(A^{*} ; C_{0}, \ldots, C_{m-1}\right)$ describe an adjoint. Then $\mathscr{P}$ is a continuous indexed operator and for the range $R(\mathscr{P})$ the following characterization is true: the data $\left(f ; g_{0}, \ldots, g_{m-1}\right) \in L^{2}(\Omega) \times X_{0}$ belong to $R(\mathscr{P})$ if and only if

$$
(f \mid v)_{0, \Omega}-\sum_{j=0}^{m-1}\left\langle g_{j} \mid T_{j} v\right\rangle_{0, \Gamma_{0}}=0
$$

for all $v \in N\left(\mathscr{P}^{*}\right)$. Thus we have $\operatorname{codim} R(\mathscr{P})=\operatorname{dim} N\left(\mathscr{P}^{*}\right)$,

$$
\varkappa(\mathscr{P})=\operatorname{dim} N(\mathscr{P})-\operatorname{dim} N\left(\mathscr{P}^{*}\right) .
$$


Note that $\mathscr{P}^{*}: H^{2 m}(\Omega) \rightarrow L^{2}(\Omega) \times X_{0}$ is also a continuous indexed operator and that $\mathscr{P}$ is an adjoint of $\mathscr{P}^{*}$ in the previous sense.

2.4. Exterior problem, assumptions. Let now $\Omega$ be an exterior domain with the smooth boundary $\Gamma_{0}$. We consider the regular boundary value problem $(2.5)$. We shall define this problem and describe its solvability in a similar general frame as was presented in the previous section for bounded domains. However, the behaviour at infinity must be taken into account.

Let us abbreviate

$$
\begin{aligned}
& H_{\mathrm{vox}}^{s}(\bar{\Omega})=\left\{u \in H^{s}(\Omega) \mid \operatorname{supp} u \text { bounded }\right\}, \\
& H_{\mathrm{loc}}^{s}(\bar{\Omega})=\left\{u \in H_{\mathrm{loc}}^{s}(\Omega) \mid \varphi u \in H^{s}(\Omega), \quad \varphi \in \mathscr{D}\left(\mathbf{R}^{n}\right)\right\}, \\
& H_{0, \mathrm{loc}}^{s}(\bar{\Omega})=\left\{u \in H_{\mathrm{loc}}^{s}(\Omega) \mid \varphi u \in H_{0}^{s}(\Omega), \quad \varphi \in \mathscr{D}\left(\mathbf{R}^{n}\right)\right\} .
\end{aligned}
$$

Here $\operatorname{supp} u$ is the support of the function $u$ and, for any open set $\tilde{\Omega} \subset \mathbf{R}^{n}, \mathscr{D}(\widetilde{\Omega})$ denotes the space of infinitely differentiable functions $\varphi$ with $\operatorname{supp} \varphi \subset \subset \widetilde{\Omega}$ (the "testfunctions of $\widetilde{\Omega}$ "). Furthermore, $H_{0}^{s}(\widetilde{\Omega})$ is the closure of $\mathscr{D}(\widetilde{\Omega})$ in $H^{s}(\widetilde{\Omega})$.

We define problem (2.5) by means of the operator

$$
\mathscr{P}: J(\Omega) \rightarrow K(\Omega) \times \prod_{j=0}^{m-1} H^{2 m-m_{j}-1 / 2}\left(\Gamma_{0}\right)=K(\Omega) \times X_{0},
$$

where $\mathscr{P}$ is defined by (2.6) and where $J(\Omega)$ and $K(\Omega)$ are given linear function spaces. The exterior problem (2.5) is now stated as the equation

$$
\mathscr{P} u=(f ; g) \text {. }
$$

In order to describe the solvability of $(2.15)$, we introduce the adjoint $\mathscr{P}^{*}$ of $\mathscr{P}$ such that

and that

$$
\mathscr{P}^{*}: J^{*}(\Omega) \rightarrow K(\Omega) \times X_{0}
$$

where the system $\left\{C_{j}\right\}_{j=0}^{m-1}$ is adjoint to $\left\{B_{j}\right\}_{j=0}^{m-1}$. The spaces $J^{(*)}(\Omega)$ and $K(\Omega)$ $\left(J^{*)}(\Omega)\right.$ denotes either $J(\Omega)$ or $\left.J^{*}(\Omega)\right)$ are required to satisfy certain conditions which are given by the following assumptions (A1)-(A6).

First, we assume that

(A1) (i) $H_{\mathrm{vox}}^{2 m}(\bar{\Omega}) \subset J^{(*)}(\Omega) \subset H_{\mathrm{loc}}^{2 m}(\bar{\Omega})$,

(ii) $K(\Omega) \subset L^{2}(\Omega)$; either $K(\Omega)$ is normed and the inclusion is continuous, or $K(\Omega)=H_{\mathrm{vox}}^{0}(\bar{\Omega})$ equipped with the locally convex topology of $H_{\mathrm{vox}}^{0}(\bar{\Omega})$.

(iii) $A u \in K(\Omega), A^{*} v \in K(\Omega)$ if $u \in J(\Omega), v \in J^{*}(\Omega)$.

In particular, assumption (A1) guarantees that the values $A u$ and $A^{*} v$ as well as the traces $B_{j} u$ and $C_{j} v$ are well defined.

An essential feature of the spaces $J^{(*)}(\Omega)$ is that they characterize the behaviour of their elements at infinity. This is the property included in the next assumption. 
(A2) For any $\varphi \in \mathscr{D}\left(\mathbf{R}^{n}\right)$ holds

$$
J^{*}(\Omega)=\left\{u \in H_{\mathrm{loc}}^{2 m}(\bar{\Omega}) \mid(1-\varphi) u \in J^{*}(\Omega)\right\} .
$$

The spaces $J^{(*)}(\Omega)$ and $K(\Omega)$ are assumed to be "dual" in the sense that we have (A3) $u \bar{f} \in L^{1}(\Omega)$ if $u \in J^{(*)}(\Omega), f \in K(\Omega)$.

In the sequel, if $u \bar{f} \in L^{1}(\Omega)$, we write

$$
(u \mid f)_{0, \Omega}:=\int_{\Omega} u \bar{f} .
$$

Furthermore, we suppose

(A4) For every $u \in J(\Omega), v \in J^{*}(\Omega)$, both vanishing in a neighbourhood of $\Gamma_{0}$,

$$
(A u \mid v)_{0, \Omega}=\left(u \mid A^{*} v\right)_{0, \Omega}
$$

is valid.

By definition of the adjoint $A^{*}$, formula (2.19) is true for all testfunctions in $\Omega$. Loosely speaking, hypothesis (A4) means that $A^{*}$ is also adjoint to $A$ with respect to the conditions at infinity included in the requirements $u \in J(\Omega), v \in J^{*}(\Omega)$.

It is worth observing that conditions (A4), (A2) imply the general Green identity

$$
(A u \mid v)_{0, \Omega}-\left(u \mid A^{*} v\right)_{0, \Omega}=\sum_{j=0}^{m-1}\left(\left\langle B_{j} u \mid T_{j} v\right\rangle_{0, \Gamma_{0}}-\left\langle S_{j} u \mid C_{j} v\right\rangle_{0, \Gamma_{0}}\right)
$$

for all $u \in J(\Omega)$ and $v \in J^{*}(\Omega)$.

Our last assumptions concern the auxiliary exterior Dirichlet problem. For every smooth exterior subdomain $\Omega_{1} \subset \Omega$, we first define

$$
\begin{aligned}
J^{*}\left(\Omega_{1}\right) & =\left\{\left.u\right|_{\Omega_{1}} \mid u \in J^{*}(\Omega)\right\}, \\
K\left(\Omega_{1}\right) & =\left\{\left.u\right|_{\Omega_{1}} \mid u \in K(\Omega)\right\} .
\end{aligned}
$$

The exterior Dirichlet problem and its adjoint are defined by means of the operators

$$
\begin{gathered}
\mathscr{P}_{1}: J\left(\Omega_{1}\right) \rightarrow K\left(\Omega_{1}\right) \times \prod_{j=0}^{m-1} H^{2 m-j-1 / 2}\left(\Gamma_{1}\right)=: K\left(\Omega_{1}\right) \times X_{1}, \\
\mathscr{P}_{1}^{*}: J^{*}\left(\Omega_{1}\right) \rightarrow K\left(\Omega_{1}\right) \times X_{1},
\end{gathered}
$$

$\Gamma_{1}=\partial \Omega_{1}$. These operators are given by

$$
\begin{gathered}
\mathscr{P}_{1} u:=\left(A u ; \gamma_{0} u, \ldots, \gamma_{m-1} u\right)=:\left(A u: \gamma^{1} u\right), \\
\mathscr{P}_{1}^{*} u:=\left(A^{*} u ; \gamma_{0} u, \ldots, \gamma_{m-1} u\right)=:\left(A^{*} u ; \gamma^{1} u\right) .
\end{gathered}
$$

Above, $\gamma_{l}$ is the trace operator $\gamma_{l} u=\left.(\partial / \partial n)^{l} u\right|_{\Gamma_{1}}$, where the normal vector $n$ is always chosen in the exterior domain.

We assume that the Dirichlet problem has certain solvability poroperties. However, we do not require that the explicit conditions given by adjoints be known. 
This will be proved as a special case in Theorem 3.6. Furthermore, we assume the knowledge of the auxiliary problem only for one exterior subdomain $\Omega_{1}$ of $\Omega$ such that $\bar{\Omega}_{1} \subset \Omega$.

In the following we shall use the term weakly indexed for linear operators $T$. By this we mean that the kernel of $T$ is finite dimensional, and that the range of $T$ has a finite codimension. The completeness of $R(T)$ will be replaced by condition (A6).

Our next assumption reads:

(A5) For a smooth exterior subdomain $\bar{\Omega}_{1} \subset \Omega$ the operators $\mathscr{P}_{1}$ and $\mathscr{P}_{1}^{*}$ are weakly indexed operators such that

$$
\chi\left(\mathscr{P}_{1}\right)=\operatorname{dim} N\left(\mathscr{P}_{1}\right)-\operatorname{codim} R\left(\mathscr{P}_{1}\right)=\operatorname{codim} R\left(\mathscr{P}_{1}^{*}\right)-\operatorname{dim} N\left(\mathscr{P}_{1}^{*}\right)=-\varkappa\left(\mathscr{P}_{1}^{*}\right) .
$$

According to (A5) there are operators $\hat{\mathscr{P}}_{1}^{-1}: K\left(\Omega_{1}\right) \times X_{1} \rightarrow J\left(\Omega_{1}\right)$ and $\left(\hat{\mathscr{P}}_{1}^{*}\right)^{-1}: K\left(\Omega_{1}\right) \times$ $X_{1} \rightarrow J^{*}\left(\Omega_{1}\right)$ with the properties

$$
\begin{gathered}
\mathscr{P}_{1} \hat{\mathscr{P}}_{1}^{-1}=Q_{1}, \quad \hat{\mathscr{P}}_{1}^{-1} \mathscr{P}_{1}=I-P_{1}, \\
\mathscr{P}_{1}^{*}\left(\hat{\mathscr{P}}_{1}^{*}\right)^{-1}=\tilde{Q}_{1}, \quad\left(\hat{\mathscr{P}}_{1}^{*}\right)^{-1} \mathscr{P}_{1}^{*}=I-\widetilde{P}_{1},
\end{gathered}
$$

where $Q_{1}$ and $\widetilde{Q}_{1}$ are projections onto the range $R\left(\mathscr{P}_{1}\right)$ and $R\left(\mathscr{P}_{1}^{*}\right)$, respectively, and where $P_{1}$ and $\tilde{P}_{1}$ are projections onto the kernels $N\left(\mathscr{P}_{1}\right)$ and $N\left(\mathscr{P}_{1}^{*}\right)$, respectively. Because of $(2.28,2.29)$, we shall use the term "pseudoinverse" for these operators (cf. Jörgens [14]).

We assume that from the (possibly many) choices of the pseudoinverses $\hat{\mathscr{P}}_{1}^{-1}$ and $\left(\hat{\mathscr{P}}_{1}^{*}\right)^{-1}$ at least one is continuous as a mapping from $K\left(\Omega_{1}\right) \times X_{1}$ into $H_{\mathrm{loc}}^{2 m}\left(\bar{\Omega}_{1}\right)$ and is such that $Q_{1}$ and $\tilde{Q}_{1}$ are continuous.

Thus, by denoting $\Omega_{1}(R)=\left\{x \in \Omega_{1}|| x \mid<R\right\}$, we require

(A6) There are pseudoinverses $\hat{\mathscr{P}}_{1}^{-1}$ and $\left(\hat{\mathscr{P}}_{1}^{*}\right)^{-1}$ such that

$$
\begin{array}{r}
\left\|\hat{\mathscr{P}}_{1}^{-1}(f ; g)\right\|_{2 m, \Omega_{1}(R)} \leqq c(R)\left(\|f\|\left(K\left(\Omega_{1}\right)\right)+\|g\|\left(X_{1}\right)\right), \\
\left\|\left(\hat{\mathscr{P}}_{1}^{*}\right)^{-1}(f ; g)\right\|_{2 m, \Omega_{1}(R)} \leqq c(R)\left(\|f\|\left(K\left(\Omega_{1}\right)\right)+\|g\|\left(X_{1}\right)\right),
\end{array}
$$

in the case where $K(\Omega)$ is normed. If $K(\Omega)=H_{\text {vox }}^{0}(\bar{\Omega})$, then $(2.30)$ and $(2.31)$ are replaced by

$$
\begin{aligned}
& \left\|\hat{\mathscr{P}}_{1}^{-1}(f ; g)\right\|_{2 m, \Omega_{1}(R)} \leqq c(R, S)\left(\|f\|_{0}+\|g\|\left(X_{1}\right)\right), \\
& \left\|\left(\hat{\mathscr{P}}_{1}^{*}\right)^{-1}(f ; g)\right\|_{2 m, \Omega_{1}(R)} \leqq c(R, S)\left(\|f\|_{0}+\|g\|\left(X_{1}\right)\right)
\end{aligned}
$$

for all $(f ; g) \in K\left(\Omega_{1}\right) \times X_{1}$ such that $\Omega_{1} \cap \operatorname{supp} f \subset \Omega_{1}(S)$.

As an illustration we mention the case of the Poisson equation

$$
\Delta u=f
$$


There we may choose

$$
\begin{aligned}
& K(\Omega)=\left\{f \in L^{2}(\Omega) \mid(1+|x|) f \in L^{2}(\Omega)\right\}, \\
& J^{(*)}(\Omega)=\left\{u \in H_{\mathrm{loc}}^{2}(\bar{\Omega}) \mid \Delta u \in K(\Omega), \nabla u \in L^{2}(\Omega)^{n},(1+|x|)^{-1} u \in L^{2}(\Omega)\right\}
\end{aligned}
$$

if the dimension of $\mathbf{R}^{n}$ is greater than two.

For the Helmholtz equation $(k>0)$

$$
\left(\Delta+k^{2}\right) u=f
$$

we may employ the spaces

$$
\begin{aligned}
& K(\Omega)=\left\{f \in L^{2}(\Omega) \mid(1+|x|) f \in L^{2}(\Omega)\right\} \\
& J(\Omega)=\left\{u \in H_{\mathrm{loc}}^{2}(\bar{\Omega}) \mid\left(\Delta+k^{2}\right) u \in K(\Omega), \frac{\partial}{\partial r} u-i k u \in L^{2}(\Omega)\right\} \\
& J^{*}(\Omega)=\left\{u \in H_{\mathrm{loc}}^{2}(\bar{\Omega}) \mid\left(\Delta+k^{2}\right) u \in K(\Omega), \frac{\partial}{\partial r} u+i k u \in L^{2}(\Omega)\right\}
\end{aligned}
$$

In both of these examples holds $N\left(\mathscr{P}_{1}\right)=N\left(\mathscr{P}_{1}^{*}\right)=\{0\}, R\left(\mathscr{P}_{1}\right)=R\left(\mathscr{P}_{1}^{*}\right)=K\left(\Omega_{1}\right) \times X_{1}$. However, there are cases where these relations have not been proved, but the above assumptions (A1)-(A6) are valid. For a more complete treatment we refer to the applications given in Chapters $4-6$.

\section{Solvability conditions}

In this section we prove a Fredholm type theorem for the exterior problem (2.5) defined by equation (2.15). The solvability condition will be stated explicitly by means of the adjoint $\mathscr{P}^{*}$.

We choose the exterior subdomain $\Omega_{1} \subset \Omega$ with the smooth boundary $\Gamma_{1}=$ $\partial \Omega_{1} \subset \Omega$. Furthermore, let $\Omega_{2} \subset \Omega$ be a smooth bounded domain such that $\mathbf{R}^{n} \backslash \Omega_{1} \subset \subset$ $\Omega_{2} \cup\left(\mathbf{R}^{n} \backslash \Omega\right)$. The domain $\Omega_{2}$ is chosen such that with $\Omega_{12}:=\Omega_{1} \cap \Omega_{2}$ the Dirichlet problem

$$
\begin{aligned}
& A u=f \in L^{2}\left(\Omega_{12}\right), \quad u \in H^{2 m}\left(\Omega_{12}\right), \\
& \left.\gamma_{j} u\right|_{\Gamma_{k}}=g_{j}^{k} \in H^{2 m-j-1 / 2}\left(\Gamma_{k}\right), j=0, \ldots, m-1, \quad k=1,2,
\end{aligned}
$$

$\Gamma_{2}=\left(\partial \Omega_{2}\right) \cap \Omega_{1}$, is uniquely solvable. If $\Omega_{1}$ has been fixed, such a choice of $\Omega_{2}$ is always possible. This follows from the strong ellipticity of $A$ and from Poincare's inequality if the width of $\Omega_{12}$ is chosen small enough.

We abbreviate $X_{2}=\prod_{j=0}^{m-1} H^{2 m-j-1 / 2}\left(\Gamma_{2}\right)$ and define the mapping

$$
\mathscr{P}_{2}: H^{2 m}\left(\Omega_{2}\right) \rightarrow L^{2}\left(\Omega_{2}\right) \times X_{0} \times X_{2}
$$

$$
\mathscr{P}_{2} u=\left(A u ; B u ; \gamma^{2} u\right)
$$


with $\gamma^{2} u=\left(\left.\gamma_{0} u\right|_{\Gamma_{2}}, \ldots,\left.\gamma_{m-1} u\right|_{\Gamma_{2}}\right)$. Let $\hat{\mathscr{P}}_{2}^{-1}$ be a Fredholm inverse (continuous pseudoinverse) of $\mathscr{P}_{2}$ such that

$$
\mathscr{P}_{2} \hat{\mathscr{P}}_{1}^{-1}=Q_{2}, \quad \hat{\mathscr{P}}_{2}^{-1} \mathscr{P}_{2}=I-P_{2},
$$

where $Q_{2}$ and $P_{2}$ are projections onto the range $R\left(\mathscr{P}_{2}\right)$ and onto the kernel $N\left(\mathscr{P}_{2}\right)$. The following result is crucial in our modification of the alternating method.

Lemma 3.1. Let $u \in J(\Omega)$ be a solution of equation (2.15). Then we have

$$
\begin{gathered}
\left.u\right|_{\Omega_{2}}=\hat{\mathscr{P}}_{2}^{-1}\left(\left.f\right|_{\Omega_{2}} ; g ; \gamma^{2} u\right)+P_{2}\left(\left.u\right|_{\Omega_{2}}\right), \\
\left.u\right|_{\Omega_{1}}=\hat{\mathscr{P}}_{1}^{-1}\left(\left.f\right|_{\Omega_{1}} ; \gamma^{1} u\right)+P_{1}\left(\left.u\right|_{\Omega_{1}}\right), \\
\gamma^{2}\left(\hat{\mathscr{P}}_{1}^{-1}\left(\left.f\right|_{\Omega_{1}} ; \gamma^{1} \hat{\mathscr{P}}_{2}^{-1}\left(\left.f\right|_{\Omega_{2}} ; g ; \gamma^{2} u\right)+\gamma^{1} P_{2}\left(\left.u\right|_{\Omega_{2}}\right)\right)+\gamma^{2} P_{1}\left(\left.u\right|_{\Omega_{1}}\right)=\gamma^{2} u .\right.
\end{gathered}
$$

Conversely, if there are the functions $(h ; w ; v) \in X_{2} \times N\left(\mathscr{P}_{1}\right) \times N\left(\mathscr{P}_{2}\right)$ such that with $(f ; g) \in K(\Omega) \times X_{0}$ the relations

$$
\begin{gathered}
\left(\left.f\right|_{\Omega_{2}} ; g ; h\right) \in R\left(\mathscr{P}_{2}\right), \\
\left(\left.f\right|_{\Omega_{1}} ; \gamma^{1} \hat{\mathscr{P}}_{2}^{-1}\left(\left.f\right|_{\Omega_{2}} ; g ; h\right)+\gamma^{1} v\right) \in R\left(\mathscr{P}_{1}\right), \\
\gamma^{2} \hat{\mathscr{P}}_{1}^{-1}\left(\left.f\right|_{\Omega_{1}} ; \gamma^{1} \hat{\mathscr{P}}_{2}^{-1}\left(\left.f\right|_{\Omega_{2}} ; g ; h\right)+\gamma^{1} v\right)+\gamma^{2} w=h
\end{gathered}
$$

are valid, then we have $(f ; g) \in R(\mathscr{P})$ and a solution $u$ of $(2.15)$ can be given by

$$
u=\left\{\begin{array}{lll}
u_{2}, & \text { in } \Omega_{2}, \\
u_{1}, & \text { in } \Omega_{1}
\end{array}\right.
$$

where the functions

$$
\begin{aligned}
u_{2} & :=\hat{\mathscr{P}}_{2}^{-1}\left(\left.f\right|_{\Omega_{2}} ; g ; h\right)+v, \\
u_{1} & :=\hat{\mathscr{P}}_{1}^{-1}\left(\left.f\right|_{\Omega_{1}} ; \gamma^{1} u_{2}\right)+w
\end{aligned}
$$

coincide in $\Omega_{12}$.

Proof. Suppose that $u \in J(\Omega)$ satisfies $\mathscr{P} u=(f ; g)$. Then we conclude $\left.u\right|_{\Omega_{2}} \in H^{2 m}\left(\Omega_{2}\right)$ and

$$
\left(\left.f\right|_{\Omega_{2}} ; g ; \gamma^{2} u\right)=\left(A\left(\left.u\right|_{\Omega_{2}}\right) ; B\left(\left.u\right|_{\Omega_{2}}\right) ; \gamma^{2}\left(\left.u\right|_{\Omega_{2}}\right)\right) \in R\left(\mathscr{P}_{2}\right) .
$$

Similarly, $\left.u\right|_{\Omega_{1}} \in J\left(\Omega_{1}\right)$ with

$$
\left(\left.f\right|_{\Omega_{1}} ; \gamma^{1} u\right)=\left(A\left(\left.u\right|_{\Omega_{1}}\right) ; \gamma^{1}\left(\left.u\right|_{\Omega_{1}}\right)\right) \in R\left(\mathscr{P}_{1}\right) .
$$

Using properties (3.4) and (2.28) of the pseudoinverses $\hat{\mathscr{P}}_{2}^{-1}$ and $\hat{\mathscr{P}}_{1}^{-1}$ we can write (3.14) and (3.15) equivalently as (3.5) and (3.6), respectively. When we take a trace, (3.5) and (3.6) yield formula (3.7).

Let us assume conversely that we have $(f ; g) \in K(\Omega) \times X_{0}$ such that for a triple $(h, w, v) \in X_{2} \times N\left(\mathscr{P}_{1}\right) \times N\left(\mathscr{P}_{2}\right)$ equations (3.8)-(3.10) are valid. 
We show first that the functions $u_{1}$ and $u_{2}$ defined by (3.12) and (3.13) coincide in $\Omega_{12}$. Define $v_{1}=\left.u_{1}\right|_{\Omega_{12}}$ and $v_{2}=\left.u_{2}\right|_{\Omega_{12}}$. Now, we obtain $v_{1}, v_{2} \in H^{2 m}\left(\Omega_{12}\right)$ together with

$$
\begin{aligned}
& A v_{2}=\left.f\right|_{\Omega_{12}}, \\
& \gamma^{2} v_{2}=\gamma^{2} u_{2}=\gamma^{2}\left(\hat{\mathscr{P}}_{2}^{-1}\left(\left.f\right|_{\Omega_{2}} ; g ; h\right)\right)+\gamma^{2} v=h, \\
& \gamma^{1} v_{2}=\gamma^{1} u_{\cdot 2},
\end{aligned}
$$

and, by (3.10), together with

$$
\begin{aligned}
& A v_{1}=\left.f\right|_{\Omega_{12}}, \\
& \gamma^{1} v_{1}=\gamma^{1} u_{2}, \\
& \gamma^{2} v_{1}=\gamma^{2} u_{1}=\gamma^{2}\left(\hat{\mathscr{P}}_{1}^{-1}\left(\left.f\right|_{\Omega_{1}} ; \gamma^{1}\left(\hat{\mathscr{P}}_{2}^{-1}\left(\left.f\right|_{\Omega_{1}} ; g ; h\right)\right)+\gamma^{1} v\right)\right)+\gamma^{2} w=h .
\end{aligned}
$$

By the unique solvability of the Dirichlet problem (3.1) we obtain from (3.16), (3.17) the assertion $v_{1}=v_{2}$.

Since the functions $u_{1}$ and $u_{2}$ coincide in $\Omega_{12}$, the function $u$ given by (3.11) is well defined. We verify that $u \in J(\Omega)$. Let $\xi \in \mathscr{D}\left(\mathbf{R}^{n}\right)$ be a testfunction such that $\xi(x) \equiv 1$ in an open set containing $\mathbf{R}^{n} \backslash \Omega_{1}$ and such that the support of $\xi$ is included in $\Omega_{2} \cup\left(\mathbf{R}^{n} \backslash \Omega_{1}\right)$.

Defining

$$
\tilde{u}_{2}(x)= \begin{cases}\xi(x) u_{2}(x), & x \in \Omega_{2}, \\ 0, & x \in \Omega \backslash \Omega_{2},\end{cases}
$$

and

$$
\tilde{u}_{1}(x)= \begin{cases}(1-\xi(x)) u_{1}(x), & x \in \Omega_{1}, \\ 0, & x \in \Omega \backslash \Omega_{1},\end{cases}
$$

we have the representation

$$
u=\tilde{u}_{1}+\tilde{u}_{\varepsilon}
$$

Since $\xi u_{1} \in H_{\text {vox }}^{2 m}\left(\bar{\Omega}_{1}\right) \subset J\left(\Omega_{1}\right)$, one concludes

$$
\tilde{u}_{1 \mid \Omega_{1}}=u_{1}-\xi u_{1} \in J\left(\Omega_{1}\right),
$$

which by (2.18), (2.21) yields

$$
\tilde{u}_{1} \in J(\Omega) \text {. }
$$

Furthermore,

$$
\tilde{u}_{2} \in H_{\text {vox }}^{2 m}(\bar{\Omega}) \subset J(\Omega) .
$$

By (3.20)-(3.22) we have $u \in J(\Omega)$. From the construction it follows that

$$
A u=f, \quad B u=g
$$

and therefore $\mathscr{P} u=(f ; g)$. This ends the proof. 
We define the linear operators $L_{j}, j=0,1,2$, letting

$$
\begin{gathered}
L_{0}(f ; g):=\gamma^{2} \hat{\mathscr{P}}_{1}^{-1}\left(\left.f\right|_{\Omega_{1}} ; \gamma^{1} \hat{\mathscr{P}}_{2}^{-1}\left(\left.f\right|_{\Omega_{2}} ; g ; 0\right)\right), \\
L_{1}(f ; g):=\left(I-Q_{1}\right)\left(\left.f\right|_{\Omega_{1}} ; \gamma^{1} \hat{\mathscr{P}}_{2}^{-1}\left(\left.f\right|_{\Omega_{2}} ; g ; 0\right)\right), \\
L_{2}(f ; g):=\left(I-Q_{2}\right)\left(\left.f\right|_{\Omega_{2}} ; g ; 0\right) .
\end{gathered}
$$

Accordingly, these operators map as follows: $L_{0}: K(\Omega) \times X_{0} \rightarrow X_{2}, L_{1}: K(\Omega) \times X_{0} \rightarrow Y_{1}$ and $L_{2}: K(\Omega) \times X_{0} \rightarrow Y_{2}$. Here $Y_{1}$ and $Y_{2}$ are the finite dimensional spaces $Y_{1}=$ $=\left(I-Q_{1}\right)\left(K\left(\Omega_{1}\right) \times X_{1}\right)$ and $Y_{2}=\left(I-Q_{2}\right)\left(L^{2}\left(\Omega_{2}\right) \times X_{0} \times X_{2}\right)$. In particular, $\operatorname{dim} Y_{1}=$ $\operatorname{codim} R\left(P_{1}\right), \operatorname{dim} Y_{2}=\operatorname{codim} R\left(P_{2}\right)$.

Conditions (3.8)-(3.10) are equivalent to the system

$$
\begin{gathered}
L_{2}(f ; g)=\left(Q_{2}-I\right)(0 ; 0 ; h), \\
L_{1}(f ; g)=\left(Q_{1}-I\right)\left(0 ; \gamma^{1} \hat{\mathscr{P}}_{2}^{-1}(0 ; 0 ; h)\right)+\left(Q_{1}-I\right)\left(0 ; \gamma^{1} v\right), \\
L_{0}(f ; g)=h-\gamma^{2} \hat{\mathscr{P}}_{1}^{-1}\left(0 ; \gamma^{1} \hat{\mathscr{P}}_{2}^{-1}(0 ; 0 ; h)\right)-\gamma^{2} \hat{\mathscr{P}}_{1}^{-1}\left(0 ; \gamma^{1} v\right)-\gamma^{2} w .
\end{gathered}
$$

Let us introduce the operators

$$
\begin{aligned}
& \Lambda_{1}: X_{2} \rightarrow X_{2}, \\
& \Lambda_{1} h=h-\gamma^{2} \hat{\mathscr{P}}_{1}^{-1}\left(0 ; \gamma^{1} \hat{\mathscr{P}}_{2}^{-1}(0 ; 0 ; h)\right) ; \\
& \Lambda_{2}: N\left(\mathscr{P}_{1}\right) \times N\left(\mathscr{P}_{2}\right) \rightarrow X_{2}, \\
& \Lambda_{2}(w, v)=-\gamma^{2} \hat{\mathscr{P}}_{1}^{-1}\left(0 ; \gamma^{1} v\right)-\gamma^{2} w ; \\
& \Lambda_{3}: X_{2} \rightarrow Y_{1} \times Y_{2}, \\
& \Lambda_{3} h=\left(\left(Q_{1}-I\right)\left(0 ; \gamma^{1} \hat{\mathscr{P}}_{2}^{-1}(0 ; 0 ; h)\right), \quad\left(Q_{2}-I\right)(0 ; 0 ; h)\right) ; \\
& \Lambda_{4}: N\left(\mathscr{P}_{1}\right) \times N\left(\mathscr{P}_{2}\right) \rightarrow Y_{1} \times Y_{2}, \\
& \Lambda_{4}(w, v)=\left(\left(Q_{1}-I\right)\left(0 ; \gamma^{1} v\right), 0\right) .
\end{aligned}
$$

Equations (3.26)-(3.28) are equivalent to

$$
\begin{aligned}
& L_{0}(f ; g)=\Lambda_{1} h+\Lambda_{2}(w, v), \\
& \left(L_{1}(f ; g), L_{2}(f ; g)\right)=\Lambda_{3} h+\Lambda_{4}(w, v) .
\end{aligned}
$$

Finally, we introduce the linear operator $\Lambda: X_{2} \times\left(N\left(\mathscr{P}_{1}\right) \times N\left(\mathscr{P}_{2}\right)\right) \rightarrow X_{2} \times\left(Y_{1} \times Y_{2}\right)$ by the matrix representation

$$
\Lambda=\left(\begin{array}{ll}
\Lambda_{1} & \Lambda_{2} \\
\Lambda_{3} & \Lambda_{4}
\end{array}\right)
$$

We have

Lemma 3.2. The kernels $N(\mathscr{P})$ and $N(\Lambda)$ are isomorphic. 
Proof. Let $(h,(w, v))$ be an element of $N(\Lambda)$. Since equations (3.33) or equivalently $(3.8)-(3.10)$ are valid with $(f ; g)=(0 ; 0)$, we may, by Lemma 3.1 , define the function $u:=T(h,(w, v)) \in J(\Omega)$ by the requirements

$$
\begin{gathered}
\left.u\right|_{\Omega_{2}}=\hat{\mathscr{P}}_{2}^{-1}(0 ; 0 ; h)+v, \\
\left.u\right|_{\Omega_{1}}=\hat{\mathscr{P}}_{1}^{-1}\left(0 ; \gamma^{1}\left(\left.u\right|_{\Omega_{2}}\right)\right)+w .
\end{gathered}
$$

By Lemma $3.1 u \in N(\mathscr{P})$. The mapping $T: N(\Lambda) \rightarrow N(\mathscr{P})$ is linear, too.

Suppose that $u=0$. Then we conclude from (3.36) that

$$
w=\left.u\right|_{\Omega_{1}}-\hat{\mathscr{P}}_{1}^{-1}\left(0 ; \gamma^{1}\left(\left.u\right|_{\Omega_{2}}\right)\right)=0
$$

By equation (3.10) then

$$
h=\gamma^{2} \hat{\mathscr{P}}_{1}^{-1}\left(0 ; \gamma^{1}\left(\left.u\right|_{\Omega_{2}}\right)\right)+\gamma^{2} w=0
$$

and (3.35), (3.38) yield $v=0$. Consequently, the mapping $T$ is injective. But it is also surjective since by the first part of Lemma 3.1 we obtain

$$
u=T\left(\gamma^{2} u,\left(P_{1}\left(\left.u\right|_{\Omega_{1}}\right), P_{2}\left(\left.u\right|_{\Omega_{2}}\right)\right)\right)
$$

if $u \in N(\mathscr{P})$. This completes the proof.

In order to prove the Fredholm alternative it is convenient to discuss the mapping $M: Z \rightarrow Z, Z:=X_{2} \times\left(N\left(\mathscr{P}_{1}\right) \times N\left(\mathscr{P}_{2}\right)\right) \times\left(Y_{1} \times Y_{2}\right)$, where $M$ is given by the matrix

$$
M=\left(\begin{array}{lll}
\Lambda_{1} & \Lambda_{2} & 0 \\
0 & 0 & 0 \\
\Lambda_{3} & \Lambda_{4} & 0
\end{array}\right) .
$$

We need the following lemma

Lemma 3.3. The mapping $h \rightarrow \gamma^{2} \hat{\mathscr{P}}_{1}^{-1}\left(0 ; \gamma^{1} \hat{\mathscr{P}}_{2}^{-1}(0 ; 0 ; h)\right), X_{2} \rightarrow X_{2}$ is compact.

Proof. The mapping $w \rightarrow \gamma^{2} \hat{\mathscr{P}}_{1}^{-1}(0 ; w), X_{1} \rightarrow X_{2}$ is continuous by the continuity of $\gamma^{2}: H_{\text {loc }}^{2 m}\left(\bar{\Omega}_{1}\right) \rightarrow X_{2}$ and by assumption (A6), which states that the pseudoinverse $\hat{\mathscr{P}}_{1}^{-1}: K\left(\Omega_{1}\right) \times X_{1} \rightarrow H_{\mathrm{loc}}^{2 m}\left(\bar{\Omega}_{1}\right)$ is continuous.

Accordingly, it is enough to show that the mapping $h \rightarrow \gamma^{1} \hat{\mathscr{P}}_{2}^{-1}(0 ; 0 ; h)$ is a compact operator $X_{2} \rightarrow X_{1}$. This property is independent of the choice of the Fredholm inverse $\hat{\mathscr{P}}_{2}^{-1}$ since two Fredholm inverses differ only by a finite dimensional continuous operator.

We choose the Fredholm inverse $\hat{\mathscr{P}}_{2}^{-1}$ in the following way. The range $R\left(\mathscr{P}_{2}\right)$ can be characterized as (Theorem 2.4):

$$
(f ; g ; h) \in R\left(\mathscr{P}_{2}\right) \text { if and only if }(f ; g ; h) \in L^{2}\left(\Omega_{2}\right) \times X_{0} \times X_{2}=: Z_{2}
$$

such that

$$
(f \mid v)_{0, \Omega_{2}}-\sum_{j=0}^{m-1}\left(\left\langle g_{j} \mid T_{j} v\right\rangle_{0, \Gamma_{0}}+\left\langle h_{j} \mid \beta_{j} v\right\rangle_{0, \Gamma_{2}}\right)=0
$$


for every $v \in N\left(\mathscr{P}_{2}^{*}\right)$. Here $\mathscr{P}_{2}^{*}$ denotes the adjoint operator $\mathscr{P}_{2}^{*} u=\left(A^{*} u ; C u ; \gamma^{2} u\right)$ and the operators $T_{j}, \beta_{j}$ are chosen such that $\left(C_{0}, \ldots, C_{m-1} ; T_{0}, \ldots, T_{m-1}\right)$ and $\left(\gamma_{0}, \ldots, \gamma_{m-1} ; \beta_{0}, \ldots, \beta_{m-1}\right)$ are Dirichlet systems of order $2 m$ on $\Gamma_{0}$ and on $\Gamma_{2}$. According to (3.39) we may use the direct decomposition

$$
Z_{2}=R\left(\mathscr{P}_{2}\right) \oplus Y_{2},
$$

where

$$
Y_{2}=\left\{(v ;-C v ;-\beta v) \mid v \in N\left(\mathscr{P}_{2}^{*}\right)\right\}
$$

with $\beta=\left(\beta_{0}, \ldots, \beta_{m-1}\right)$.

Take $(0 ; 0 ; h) \in Z_{2}$ and write $w=\hat{\mathscr{P}}_{2}^{-1}(0 ; 0 ; h)$. Then we obtain by $(3.4)$

$$
\mathscr{P}_{2} w=Q_{2}(0 ; 0 ; h)=:(\tilde{f} ; \tilde{g} ; \tilde{h}) .
$$

Since the kernel $N\left(\mathscr{P}^{*}\right)$ contains only smooth functions in $\bar{\Omega}_{2}$, we conclude $\tilde{f} \in C^{\infty}\left(\bar{\Omega}_{2}\right), g \in C^{\infty}\left(\Gamma_{0}\right)$ and

$$
\begin{gathered}
\|\tilde{f}\|_{s, \Omega_{2}} \leqq c(s)\|h\|\left(X_{2}\right), \\
\|\tilde{g}\|_{\tau, \Gamma_{0}} \leqq c(\tau)\|h\|\left(X_{2}\right), \\
\|\tilde{h}\|\left(X_{2}\right) \leqq c\|h\|\left(X_{2}\right) .
\end{gathered}
$$

From the interior regularity results for elliptic operators it follows by (3.42) that $w \in H^{2 m+1}(\tilde{\tilde{\Omega}})$ with

$$
\|w\|_{2 m+1, \tilde{\tilde{\Omega}}} \leqq c\left(\|\tilde{f}\|_{1, \tilde{\Omega}}+\|w\|_{0, \Omega_{2}}\right)
$$

for interior subdomains $\tilde{\tilde{\Omega}} \subset \subset \tilde{\Omega} \subset \subset \Omega_{2}$. By (3.43), (3.44) and by the continuity of $\hat{\mathscr{P}}_{2}^{-1}$ we obtain

$$
\|w\|_{2 m+1, \tilde{\Omega}} \leqq c\|h\|\left(X_{2}\right) .
$$

From (3.45) it follows that $h \rightarrow \hat{\mathscr{P}}_{2}^{-1}(0 ; 0 ; h): X_{2} \rightarrow H^{2 m}(\tilde{\tilde{\Omega}})$ is compact, which yields the assertion by the continuity of the trace $\gamma^{1}: H_{\mathrm{loc}}^{2 m}\left(\Omega_{2}\right) \rightarrow X_{1}$.

In the sequel the space $X_{2}$ as well as the finite dimensional spaces $N\left(\mathscr{P}_{i}\right)$, $Y_{i}$ and their products are considered as Hilbert spaces. The appearing adjoints and orthogonality conditions are thus well defined.

We define $L: K(\Omega) \times X_{0} \rightarrow X_{2} \times Y_{1} \times Y_{2}$ by

$$
L(f ; g)=\left(L_{0}(f ; g) ; L_{1}(f ; g) ; L_{2}(f ; g)\right) .
$$

Furthermore, we write $\beta_{j}=\operatorname{codim} R\left(\mathscr{P}_{j}\right), \alpha_{j}=\operatorname{dim} N\left(\mathscr{P}_{j}\right)$ and for the index of $\mathscr{P}_{j}$

$$
\varkappa_{j}=\alpha_{j}-\beta_{j},
$$

$j=1,2$. For $\mathscr{P}$ we use the notation $x=\alpha-\beta, \alpha=\operatorname{dim} N(\mathscr{P}), \beta=\operatorname{codim} R(\mathscr{P})$.

Lemma 3.4. The following assertions are true.

(a) The operator $M$ is of the form $M=I-K, K$ compact. 
(b) The kernel $N(\mathscr{P})$ is finite dimensional and we have

$$
\alpha=\operatorname{dim} N(M)-\beta_{1}-\beta_{2} .
$$

(c) For the range $R(\mathscr{P})$ we have

$$
R(\mathscr{P})=\left\{(f ; g) \in K(\Omega) \times X_{0} \mid L(f ; g) \perp N\left(\Lambda^{*}\right)\right\},
$$

where the kernel $N\left(\Lambda^{*}\right)$ has the dimension

$$
\operatorname{dim} N\left(\Lambda^{*}\right)=\alpha-\varkappa_{1}-\varkappa_{2} .
$$

Proof. According to the previous lemma we have $\Lambda_{1}=I-K_{1}$, where $K_{1}$ is a compact operator. This implies assertion (a) as the spaces $N\left(\mathscr{P}_{1}\right) \times N\left(\mathscr{P}_{2}\right)$ and $Y_{1} \times Y_{2}$ are finite dimensional.

Assertion (b) is a consequence of the formula

$$
N(M)=N(\Lambda) \times\left(Y_{1} \times Y_{2}\right)
$$

together with Lemma 3.2 and the fact that the kernel $N(M)=N(I-K)$ is finite dimensional by the compactness of $K$.

By equation (3.33), the condition $(f ; g) \in R(\mathscr{P})$ can be written as

$$
\left(L_{0}(f ; g) ; 0 ;\left(L_{1}(f ; g), L_{2}(f ; g)\right)\right) \in R(M) .
$$

By the Fredholm properties of $M=I-K$, the requirement (3.51) is equivalent to

$$
\left(L_{0}(f ; g) ; 0 ;\left(L_{1}(f ; g), L_{2}(f: g)\right)\right) \perp N\left(M^{*}\right),
$$

where $M^{*}: Z \rightarrow Z$ is given by

$$
M^{*}=\left(\begin{array}{rrl}
\Lambda_{1}^{*} & 0 & \Lambda_{3}^{*} \\
\Lambda_{2}^{*} & 0 & \Lambda_{4}^{*} \\
0 & 0 & 0
\end{array}\right) .
$$

Condition (3.52) reads

$$
\left(L_{0}(f ; g) ;\left(L_{1}(f ; g), L_{2}(f ; g)\right)\right) \perp N\left(\Lambda^{*}\right),
$$

where the operator $\Lambda^{*}: X_{2} \times\left(Y_{1} \times Y_{2}\right) \rightarrow X_{2} \times\left(N\left(\mathscr{P}_{1}\right) \times N\left(\mathscr{P}_{2}\right)\right)$ is defined by

$$
\Lambda^{*}=\left(\begin{array}{cc}
\Lambda_{1}^{*} & \Lambda_{3}^{*} \\
\Lambda_{2}^{*} & \Lambda_{4}^{*}
\end{array}\right) .
$$

We have $N\left(M^{*}\right)=\left\{\left(g_{1} ; g_{2} ; g_{3}\right) \mid\left(g_{1} ; g_{3}\right) \in N\left(\Lambda^{*}\right), \quad g_{2} \in N\left(\mathscr{P}_{1}\right) \times N\left(\mathscr{P}_{2}\right)\right\}, \quad$ which by (3.48) yields

$$
\operatorname{dim} N\left(\Lambda^{*}\right)=\operatorname{dim} N\left(M^{*}\right)-\left(\alpha_{1}+\alpha_{2}\right)=\operatorname{dim} N(M)-\left(\alpha_{1}+\alpha_{2}\right)=\alpha-\varkappa_{1}-\varkappa_{2} .
$$

Our aim is to express the solvability by using the adjoint problem defined by means of $\mathscr{P}^{*}=\left(A^{*} ; C\right)$. We note first 
Lemma 3.5. Let $u \in J(\Omega)$ with $\mathscr{P} u=(f ; g)$ be given. Then we have the orthogonality relation

for every $v \in N\left(\mathscr{P}^{*}\right)$.

$$
(f \mid v)_{0, \Omega}-\sum_{j=0}^{m-1}\left\langle g_{j} \mid T_{j} v\right\rangle_{0, \Gamma_{0}}=0
$$

Proof. This follows from the general Green formula (2.20).

Finally, we get our main result for the solvability of the exterior boundary value problem (2.15).

Theorem 3.6. Let $\Omega \subset \mathbf{R}^{n}$ be an exterior domain with a smooth boundary $\Gamma_{0}$. Furthermore, let $A$ be a strongly elliptic operator in $\Omega$ and let $\left\{B_{j}\right\}_{j=0}^{m-1}$ be a system of the boundary operators $B_{j}$ on $\Gamma_{0}$ such that problem (2.5) is regular. If assumptions (A1)-(A6) are valid, then the operator $\mathscr{P}: J(\Omega) \rightarrow K(\Omega) \times$ $\prod_{j=0}^{m-1} H^{2 m-m_{j}-1 / 2}\left(\Gamma_{0}\right), \quad \mathscr{P} u=\left(A u ; B_{0} u, \ldots, B_{m-1} u\right)$, which describes the exterior problem (2.15), has the finite index $\varkappa_{1}+\varkappa_{1} \varkappa_{2}$, where $\varkappa_{i}$ is the index of the auxiliary operator $\mathscr{P}_{i}$.

Solvability conditions read: the data $(f ; g) \in K(\Omega) \times \prod_{j=0}^{m-1} H^{2 m-m_{j}-1 / 2}\left(\Gamma_{0}\right)$ belong to the range $R(\mathscr{P})$ if and only if equation (3.56) is valid for all $v \in N\left(\mathscr{P}^{*}\right)$.

Proof. We first show the inequality

$$
\operatorname{dim} N\left(\mathscr{P}^{*}\right) \leqq \operatorname{dim} N\left(\Lambda^{*}\right) .
$$

Take a testfunction $\varphi \in \mathscr{D}\left(\mathbf{R}^{n}\right), \varphi \geqq 0$ and consider the mapping $R: N\left(\mathscr{P}^{*}\right) \rightarrow N\left(\Lambda^{*}\right)$

$$
R v=\Pi L(\varphi v ; 0),
$$

where $\Pi$ is the orthogonal projection of $X_{2} \times\left(Y_{1} \times Y_{2}\right)$ onto $N\left(\Lambda^{*}\right)$. Since the space $N\left(\mathscr{P}^{*}\right)$ is finite dimensional (for the same reason as $N(\mathscr{P})$ ), we may choose the support $\varphi$ so large that for all $v \in N\left(\mathscr{P}^{*}\right)$ holds

$$
\int_{\Omega} \varphi|v|^{2}=0
$$

if and only if $v=0$. But then the mapping $R$ is injective since from $R v=0$ it follows that $\Pi L(\varphi v ; 0)=0$, which means $L(\varphi v ; 0) \in N\left(\Lambda^{*}\right)^{\perp}$. Accordingly, by Lemma 3.4 (c), there exists a solution $u$ of the equation $\mathscr{P} u=(\varphi v ; 0)$. The necessary solvability condition (3.56) then yields

implying $v=0$.

$$
0=(\varphi v \mid v)_{0, \Omega}=\int_{\Omega} \varphi|v|^{2}
$$

Thus (3.57) is proved. Combining (3.57) and (3.50) we obtain

$$
\operatorname{dim} N\left(\mathscr{P}^{*}\right) \leqq \operatorname{dim} N\left(\Lambda^{*}\right)=\operatorname{dim} N(\mathscr{P})-\varkappa_{1}-\varkappa_{2} .
$$

Changing the roles of $\mathscr{P}$ and $\mathscr{P}^{*}$ one concludes

$$
\operatorname{dim} N(\mathscr{P}) \leqq \operatorname{dim} N\left(\mathscr{P}^{*}\right)+\varkappa_{1}+\varkappa_{2} .
$$


Inequalities (3.60) and (3.61) imply

$$
\operatorname{dim} N(\mathscr{P})-\operatorname{dim} N\left(\mathscr{P}^{*}\right)=\varkappa_{1}+\varkappa_{2} .
$$

It remains to show that $\operatorname{dim} N\left(\mathscr{P}^{*}\right)=\operatorname{codim} R(\mathscr{P})$, that is, that condition (3.56) is also sufficient. Let $(f ; g) \in K(\Omega) \times X_{0}$, such that (3.56) holds, be given. Comparing formulae (3.50) and (3.62) we observe that $\operatorname{dim} N\left(\mathscr{P}^{*}\right)=\operatorname{dim} N\left(\Lambda^{*}\right)$. Accordingly, the mapping $R$ also is bijective. Hence we find an element $v \in N\left(\mathscr{P}^{*}\right)$ such that

$$
\Pi L(f-\varphi v ; g)=0 .
$$

But Lemma 3.5 and characterization (3.49) then yield

$$
(f-\varphi v \mid v)_{0, \Omega}-\sum_{j=0}^{m-1}\left\langle g_{j} \mid T_{j} v\right\rangle_{0, \Gamma_{0}}=0 .
$$

By condition (3.56) and formula (3.64)

$$
(\varphi v \mid v)_{0, \Omega}=0
$$

which implies $\varphi v=0$. But (3.63) then implies $\Pi L(f ; g)=0$, which according to Lemma 3.4 means that $(f ; g) \in R(\mathscr{P})$.

\section{Potential type equations}

In this section we apply Theorem 3.6 to the case where the coefficients $a_{x \beta}$ of the operator

$$
A=\sum_{0 \leqq|\alpha|,|\beta| \leqq m}(-1)^{|\alpha|} \partial^{\alpha}\left(a_{\alpha \beta} \partial^{\beta}\right)
$$

not belonging to the principal part $|\alpha|=|\beta|=m$ fall to zero sufficiently rapidly at infinity. A typical example is the iterated Laplacian $A=\Delta^{m}$.

In order to discuss the general exterior boundary value problem we introduce some appropriate weighted Sobolev spaces. The weights will be chosen such that the Dirichlet sesquilinear form

$$
B(u, v)=\sum_{0 \leqq|\alpha|,|\beta| \leqq m}\left(a_{\alpha \beta} \partial^{\beta} u \mid \partial^{\alpha} v\right)_{0, \Omega}
$$

associated with the operator $A$ becomes, under certain assumptions on the coefficients, coercive for the Dirichlet problem. This will be achieved by estimating the terms which do not belong to the principal part by means of the weighted Poincaré inequalities of the type

$$
\sum_{|\alpha|=j}\left\|\frac{\partial^{\alpha} u}{(1+|x|)^{\tau} \ln (e+|x|)^{\delta}}\right\|_{0, \Omega}^{2} \leqq c|u|_{m, \Omega}^{2}
$$

for $0 \leqq j \leqq m-1$ with the seminorm $|\cdot|_{m, \Omega}$ such that

$$
|u|_{m, \Omega}^{2}=\sum_{|\alpha|=m}\left\|\partial^{\alpha} u\right\|_{0, \Omega}^{2} .
$$


Estimates (4.3) are first proved in the space $\mathscr{D}(\Omega)$. The parameters $\tau$ and $\delta$, where $\tau \geqq 0$ and $\delta=0$ or 1 , will be dependent on $m$ and $j$ as well as on the space dimension $n$.

We begin by considering exterior domains $\Omega$ such that the origin $x=0$ does not belong to $\Omega$.

Lemma 4.1. Let $\Omega \subset \mathbf{R}^{n}, n \geqq 2$ be an exterior domain such that $x \neq 0$ for all $x \in \Omega$. The following three assertions are true.

(i) If $\tau \in \mathbf{R}, \tau \neq 0$ and if $n \neq 2 \tau$, then we have for all $u \in \mathscr{D}(\Omega)$ the estimate

$$
\left\|\frac{u}{|x|^{\tau}}\right\|_{0, \Omega} \leqq 2|2 \tau-n|^{-1}\left\|\frac{\nabla u}{|x|^{\tau-1}}\right\|_{0, \Omega} .
$$

(ii) Let $|x| \geqq e$ for all $x \in \Omega$. If $2 \tau-n>0$, we have

for all $u \in \mathscr{D}(\Omega)$.

$$
\left\|\frac{u}{|x|^{\tau} \ln |x|}\right\|_{0, \Omega} \leqq 2(2 \tau-n)^{-1}\left\|\frac{\nabla u}{|x|^{\tau-1} \ln |x|}\right\|_{0, \Omega}
$$

(iii) Let $|x| \geqslant e$ for all $x \in \Omega$. If $n=2 \tau$, it holds that

for all $u \in \mathscr{D}(\Omega)$.

$$
\left\|\frac{u}{|x|^{\tau} \ln |x|}\right\|_{0, \Omega} \leqq 2\left\|\frac{\nabla u}{|x|^{\tau-1}}\right\|_{0, \Omega}
$$

Proof. (i) A partial integration yields for every $s \in \mathbf{R}$ and $\tau \neq 0$ the identity

$$
\left\|\frac{\nabla u}{|x|^{\tau-1}}+s \frac{u}{|x|^{\tau}} \frac{x}{|x|}\right\|_{0, \Omega}^{2}=\left\|\frac{\nabla u}{|x|^{\tau-1}}\right\|_{0, \Omega}^{2}+s(s+2 \tau-n)\left\|\frac{u}{|x|^{\tau}}\right\|_{0, \Omega}^{2} .
$$

By choosing $s=n-2 \tau$ we obtain

$$
\left\|\frac{\nabla u}{|x|^{\tau-1}}+s \frac{u}{|x|^{\tau}} \frac{x}{|x|}\right\|_{0, \Omega} \leqq\left\|\frac{\nabla u}{|x|^{\tau-1}}\right\|_{0, \Omega} .
$$

Since $s=n-2 \tau \neq 0$, we have by (4.9)

$$
|s|\left\|\frac{u}{|x|^{\tau}}\right\|_{0, \Omega} \leqq\left\|\frac{\nabla u}{|x|^{\tau-1}}+s \frac{u}{|x|^{\tau}} \frac{x}{|x|}\right\|_{0, \Omega}+\left\|\frac{\nabla u}{|x|^{\tau-1}}\right\|_{0, \Omega} \leqq 2\left\|\frac{\nabla u}{|x|^{\tau-1}}\right\|_{0, \Omega},
$$

which proves (4.5).

(ii) A similar calculation as above gives

$$
\begin{aligned}
\left\|\frac{\nabla u}{|x|^{\tau-1} \ln |x|}+s \frac{u}{|x|^{\tau} \ln |x|} \frac{x}{|x|}\right\|_{0, \Omega}^{2} & =\left\|\frac{\nabla u}{|x|^{\tau-1} \ln |x|}\right\|_{0, \Omega}^{2}+s(s+2 \tau-n) \| \frac{u}{|x|^{\tau} \ln \mid \overline{x \mid} \|_{0, \Omega}^{2}} \\
& +2 s\left\|\frac{u}{|x|^{\tau}(\ln |x|)^{3 / 2}}\right\|_{0, \Omega}^{2} .
\end{aligned}
$$


Since $2 \tau-n>0$, the choice $s=n-2 \tau<0$ yields

$$
\left\|\frac{\nabla u}{|x|^{\tau-1} \ln |x|}+s \frac{u}{|x|^{\tau} \ln |x|} \frac{x}{|x|}\right\|_{0, \Omega} \leqq\left\|\frac{\nabla u}{|x|^{\tau-1} \ln |x|}\right\|_{0, \Omega},
$$

which implies the assertion as in (i).

(iii) If $n=2 \tau$, we have for all $u \in \mathscr{D}(\Omega)$

$$
\left\|\frac{\nabla u}{|x|^{\tau-1}}-\frac{u}{|x|^{\tau} \ln |x|} \frac{x}{|x|}\right\|_{0, \Omega}=\left\|\frac{\nabla u}{|x|^{\tau-1}}\right\|_{0, \Omega},
$$

which leads to estimate (4.7).

Estimates (4.5) - (4.7) are applied as follows. We introduce the weights $q_{m, j}$, $j=0,1, \ldots, m-1$, which also depend on the dimension of the underlying space. We define

$$
q_{m, j}(x)= \begin{cases}|x|^{-m+j}, & n \text { odd, or } n \text { even with } n \leqq 2 m+1 \\ |x|^{-m+j}(\ln |x|)^{-1}, & n=2 l, \quad 1 \leqq l \leqq m, 0 \leqq j \leqq m-l \\ |x|^{-m+j}, & n=2 l, \quad 1 \leqq l \leqq m, m-l<j<m\end{cases}
$$

In addition to these weights we use the weights $p_{m, j}$ which behave as $q_{m, j}$ at infinity but which have no singularity for $|x|=0$ and are thus applicable to all exterior domains.

We denote

$$
p_{m, j}(x)= \begin{cases}(1+|x|)^{-m+j}, & n \text { odd, or } n \text { even with } n \leqq 2 m+1, \\ (1+|x|)^{-m+j}(\ln (e+|x|))^{-1}, & n=2 l, 1 \leqq l \leqq m, 0 \leqq j \leqq m-l, \\ (1+|x|)^{-m+j}, & n=2 l, 1 \leqq l \leqq m, m-l<j<m .\end{cases}
$$

Now, we introduce in $\mathscr{D}(\Omega)$ the norm \|\|$_{\|, p, \Omega}$ containing a contribution of all derivatives up to order $m$.

$$
\|\| u\left\|_{m, p, \Omega}^{2}:=\sum_{j=0}^{m} \sum_{|\alpha|=j}\right\| p_{m, j} \partial^{\alpha} u \|_{0, \Omega}^{2} .
$$

If $\Omega$ is an exterior domain such that $|x| \geqq e$ for all $x \in \Omega$, we also make use of the norm

$$
\mid\|u\|_{m, q, \Omega}^{2}=\sum_{j=0}^{m} \sum_{|\alpha|=j}\left\|q_{m, j} \partial^{\alpha} u\right\|_{0, \Omega}^{2} .
$$

The seminorm $|\cdot|_{m, \Omega}$ is a norm in $\mathscr{D}(\Omega)$. In fact we can prove

Lemma 4.2. Let $\Omega \subset \mathbf{R}^{n}$ be an exterior domain with $\bar{\Omega} \neq \mathbf{R}^{n}$. Then there exists a constant $c_{1}>0$ such that

$$
c_{1}^{-1}\left|\left\|\left.u\left|\left\|_{m, p, \Omega} \leqq|u|_{m, \Omega} \leqq\right\| u\right|\right|_{m, p, \Omega}\right.\right.
$$


for all $u \in \mathscr{D}(\Omega)$. If, in addition, $|x| \geqq e$ for all $x \in \Omega$, then there exists a constant $c_{2}>0$ such that

$$
\left.c_{2}^{-1}|||u|\right|_{m, q, \Omega} \leqq|u|_{m, \Omega} \leqq\left|\|u \mid\|_{m, q, \Omega}\right.
$$

for all $u \in \mathscr{D}(\Omega)$.

Proof. Since $\bar{\Omega} \neq \mathbf{R}^{n}$, we may without loss of generality assume $|x|>e$ for any $x \in \Omega$. Hence it suffices to prove the last assertion.

Let us first assume that $n$ is odd or that $n$ is even together with $n \geqq 2 m+1$. Then we have, by using inequality (4.5) successively with $\tau=m, m-1, \ldots, 1$, the estimates

$$
\left\|\frac{u}{|x|^{m}}\right\|_{0, \Omega} \leqq c\left\|\frac{\nabla u}{|x|^{m-1}}\right\|_{0, \Omega} \leqq \ldots \leqq c|u|_{m, \Omega} .
$$

In the case $n=2 l, 1 \leqq l \leqq m$, we use $m-l$ times estimate (4.6) and one time (4.7) as well as $l-1$ times (4.5). This yields the required assertion

$$
\left\|\frac{u}{|x|^{m} \ln |x|}\right\|_{0, \Omega} \leqq \ldots \leqq c \sum_{|\alpha|=m-l}\left\|\frac{\partial^{\alpha} u}{|x|^{l} \ln |x|}\right\|_{0, \Omega} \leqq c \sum_{|x|=m-l+1}\left\|\frac{\partial^{\alpha} u}{|x|^{l-1}}\right\|_{0, \Omega} \leqq \ldots \leqq c|u|_{m, \Omega} .
$$

We remark that as in inequalities (4.5)-(4.7) it is possible to give explicit bounds for the constants $c_{1}$ and $c_{2}$ appearing in (4.18) and (4.19).

We abbreviate

$$
\mathscr{D}(\bar{\Omega})=\left\{u \in C^{\infty}(\bar{\Omega}) \mid \operatorname{supp} u \text { compact }\right\}
$$

and define the weighted spaces

$$
\begin{aligned}
& H_{w}^{m}(\Omega)=\overline{\mathscr{D}(\bar{\Omega})} \mid\|\cdot\| \cdot \|_{m, p, \Omega}, \\
& H_{0 w}^{m}(\Omega)=\overline{\mathscr{D}(\Omega)} \mid\|\cdot\| \cdot \|_{m, p, \Omega} .
\end{aligned}
$$

By Lemma 4.2 it follows that $|\cdot|_{m, \Omega}$ and $\|\left.|\cdot|\right|_{m, p, \Omega}$ are equivalent norms in the space $H_{0 w}^{m}(\Omega)$. The following result makes it possible to identify more directly which functions belong to the spaces $H_{w}^{m}(\Omega)$ and $H_{0 w}^{m}(\Omega)$.

Theorem 4.3. Let $\Omega \subset \mathbf{R}^{n}$ be an exterior domain. Then the following characterizations for the spaces $H_{w}^{m}(\Omega)$ and $H_{0 w}^{m}(\Omega)$ are true.

(i) $H_{0 w}^{m}(\Omega)=\left\{u \in H_{0, \text { loc }}^{m}(\bar{\Omega}) \mid\|u\|_{m, p, \Omega}<\infty\right\}$.

(ii) If $\Omega$ has the segment property, then

$$
H_{w}^{m}(\Omega)=\left\{u \in H_{\mathrm{loc}}^{m}(\bar{\Omega}) \mid\|u\|_{m, p, \Omega}<\infty\right\} .
$$

(iii) If the boundary $\Gamma=\partial \Omega$ is smooth, then

$$
H_{0 w}^{m}(\Omega)=\left\{u \in H_{w}^{m}(\Omega)\left|\gamma_{j} u\right|_{\Gamma}=0, \quad j=0,1, \ldots, m-1\right\} .
$$

Proof. We argue only the assertion (i). For the moment we define

$$
\tilde{H}_{0 w}^{m}(\Omega)=\left\{u \in H_{0,10 c}^{m}(\Omega) \mid\|u\| \|_{m, p, \Omega}<\infty\right\} .
$$


Then the inclusion $H_{0 w}^{m}(\Omega) \subset \tilde{H}_{0 w}^{m}(\Omega)$ is clear. On the other hand let $u \in \tilde{H}_{0 w}^{m}(\Omega)$ be given. We take a fixed smoothing function $\varphi \in \mathscr{D}(\mathbf{R})$ such that $\varphi(t)=1,|t|<1$ and $\varphi(t)=0,|t|>2$, and define the sequence $\varphi_{k} \in \mathscr{D}\left(\mathbf{R}^{n}\right)$ of the testfunctions $\varphi_{k}$, $k \in \mathbf{N}, k>e^{e}$ by

$$
\varphi_{k}(x)= \begin{cases}1, & |x|<e^{e}, \\ \varphi\left(\frac{\ln \ln |x|}{\ln \ln k}\right), & |x| \geqq e^{e}\end{cases}
$$

This sequence satisfies the estimate

$$
\left|\partial^{\tau} \varphi_{k}(x)\right| \leqq c(1+|x|)^{-|\tau|} \ln (e+|x|)^{-1},
$$

where the constant $c$ is uniform with respect to $k$.

If $u \in \tilde{H}_{0 w}^{m}(\Omega)$, then we have $u \varphi_{k} \in H_{0}^{m}(\Omega)$. Furthermore, a straightforward calculation using (4.27) yields with $E(R)=\{x|| x \mid>R\}$ the upper estimate

$$
\left\|\left.\left|u-u \varphi_{k}\right|\right|_{m, p, \Omega}=\right\| u-u \varphi_{k}\left\|_{m, p, E(k)} \leqq c\right\| u \|_{m, p, E(k)} \rightarrow 0 .
$$

This proves the assertion.

The spaces $K(\Omega), J(\Omega)$ and $J^{*}(\Omega)$ which are needed for the exact definition of the exterior problem

$$
\begin{aligned}
& A u=f, \\
& B_{j} u=g_{j}, j=0,1, \ldots, m
\end{aligned}
$$

are chosen as follows. We take

$$
K(\Omega)=\left\{f \in L^{2}(\Omega) \mid\left(p_{m, 0}\right)^{-1} f \in L^{2}(\Omega)\right\},
$$

where the space $K(\Omega)$ is endowed with the norm

$$
\|f\|(K(\Omega))=\left\|\left(p_{m, 0}\right)^{-1} f\right\|_{0, \Omega} .
$$

For $J(\Omega)$ and $J^{*}(\Omega)$ we choose

$$
\begin{gathered}
J(\Omega)=\left\{u \in H_{\mathrm{loc}}^{2 m}(\bar{\Omega}) \cap H_{w}^{m}(\Omega) \mid A u \in K(\Omega)\right\}, \\
J^{*}(\Omega)=\left\{u \in H_{\mathrm{loc}}^{2 m}(\bar{\Omega}) \cap H_{w}^{m}(\Omega) \mid A^{*} u \in K(\Omega)\right\} .
\end{gathered}
$$

Now we turn to the general assumptions of Section 2.4. The assumptions (A1)-(A3) are clearly valid. Furthermore, let $u \in J(\Omega)$ and $v \in J^{*}(\Omega)$ such that $u$ and $v$ vanish in a neighbourhood of $\Gamma_{0}$. Then we have

$$
(A u \mid \varphi)_{0, \Omega}=B(u, \varphi)
$$

for all $\varphi \in \mathscr{D}(\Omega)$ and

$$
\left(A^{*} v \mid \psi\right)_{0, \Omega}=\overline{B(\psi, v)}
$$

for all $\psi \in \mathscr{D}(\Omega)$. To employ the relations (4.33) and (4.34) for $\varphi \in J^{*}(\Omega), \psi \in J(\Omega)$ we have to impose such conditions on the coefficients $a_{\alpha \beta}$ that the sesquilinear form $B$ becomes continuous with respect of $\varphi$ and $\psi$ in these spaces. 
We make the assumption that for all multi-indices $\alpha, \beta,|\alpha|,|\beta| \leqq m$,

$$
\left|a_{\alpha \beta}(x)\right| \leqq c p_{m,|\alpha|}(x) \cdot p_{m,|\beta|}(x), \quad x \in \Omega,
$$

with a positive constant $c$.

If (B1) is valid, then the sesquilinear form $B(.,$.$) is well defined and continuous$ in $H_{w}^{m}(\Omega) \times H_{w}^{m}(\Omega)$.

Furthermore, if $u \in J(\Omega) \cap H_{0 w}^{m}(\Omega), v \in J^{*}(\Omega) \cap H_{0 w}^{m}(\Omega)$, there exist sequences $\varphi_{v}, \psi_{v} \in \mathscr{D}(\Omega), \psi_{v} \rightarrow v$ in $H_{0 w}^{m}(\Omega)$ and $\varphi_{v} \rightarrow u$ in $H_{0 w}^{m}(\Omega)$. Accordingly, by (4.33) and (4.34),

$$
\begin{gathered}
(A u \mid v)_{0 \Omega}=\lim _{v}\left(A u \mid \psi_{v}\right)_{0, \Omega}=\lim _{v} B\left(u, \psi_{v}\right)=B(u, v), \\
\left(u \mid A^{*} v\right)_{0, \Omega}=\lim _{v}\left(\varphi_{v} \mid A^{*} v\right)_{0, \Omega}=\lim _{v} B\left(\varphi_{v}, v\right)=B(u, v) .
\end{gathered}
$$

Thus, if condition (B1) is valid, then (A4) also holds.

For convenience, we finally assume that the sesquilinear form $B(.,$.$) is strongly$ coercive in $H_{0 w}^{m}\left(\Omega_{1}\right)$ for the exterior subdomain $\Omega_{1}=\left\{x|| x \mid>R_{1}\right\}$. This means

(B2) There exists a constant $c_{1}>0$ such that

$$
c_{1}\||u|\|_{m, p, \Omega_{1}}^{2} \leqq \operatorname{Re} B(u, u)
$$

for all $u \in H_{0 w}^{m}\left(\Omega_{1}\right)$.

This assumption is valid if for example the coefficients $a_{\alpha \beta}$ with $|\alpha|+|\beta|<2 m$ are "small enough" and if $A$ is uniformly strongly elliptic in $\Omega$. If namely

$$
\left|a_{\alpha \beta}(x)\right| \leqq \varepsilon p_{m,|\alpha|}(x) p_{m,|\beta|}(x)
$$

for all $|\alpha|+|\beta|<2 m$, inequality (B2) is valid if $0 \leqq \varepsilon \leqq \varepsilon_{0}$ when $\varepsilon_{0}>0$ is sufficiently small. For this we write $B=B_{0}+B_{1}$, where

By (4.18) and (4.38) we have

$$
\begin{aligned}
& B_{0}(u, v)=\sum_{|\alpha|=|\beta|=m}\left(a_{\alpha \beta} \partial^{\beta} u \mid \partial^{\alpha} v\right)_{0, \Omega_{1}}, \\
& B_{1}(u, v)=\sum_{|\alpha|+|\beta|<2 m}\left(a_{\alpha \beta} \partial^{\beta} u \mid \partial^{\alpha} v\right)_{0, \Omega_{1}} .
\end{aligned}
$$

$$
\left|B_{1}(u, u)\right| \leqq c \varepsilon|u|_{m, \Omega_{1}}^{2} .
$$

Then the uniform strong ellipticity implies

$$
\operatorname{Re} B(u, u) \geqq a_{0}|u|_{m, \Omega_{1}}^{2}-c \varepsilon|u|_{m, \Omega_{1}}^{2} \geqq \frac{a_{0}}{2}|u|_{m, \Omega_{1}}^{2}
$$

if $0 \leqq \varepsilon \leqq c^{-1} a_{0} / 2$.

We collect our conclusions concerning the general assumption of Section 2.4.

Lemma 4.4. Let $A$ be a strongly uniformly elliptic operator defined by (4.1) such that the coefficients are smooth and that they satisfy the conditions (B1), (B2). If the spaces $K(\Omega), J(\Omega), J^{*}(\Omega)$ are defined by (4.30)-(4.32), then the assumptions (A1)-(A6) are valid. Furthermore it holds that $N\left(\mathscr{P}_{1}\right)=N\left(\mathscr{P}_{1}^{*}\right)=\{0\}, \quad R\left(\mathscr{P}_{1}\right)=$ $R\left(\mathscr{P}_{1}^{*}\right)=K\left(\Omega_{1}\right) \times X_{1}$. 
Proof. It remains to prove the conditions (A5) and (A6). Let $u \in N\left(\mathscr{P}_{1}\right)$. Then we have

$$
0=\operatorname{Re}(A u \mid u)_{0, \Omega_{1}}=\operatorname{Re} B(u, u) \geqq c\|\| u \|_{m, p, \Omega_{1}}^{2},
$$

which implies $u=0$. In the same way $N\left(\mathscr{P}_{1}^{*}\right)=\{0\}$.

Let on the other hand $(f ; g) \in K\left(\Omega_{1}\right) \times X_{1}$ be given. We construct the solution $w$ as follows. By Poincare's inequality the form $B(u, v)$ is strongly coercive in $H_{0}^{m}\left(B\left(R_{1}, R_{2}\right)\right)$ if $R_{2}=R_{1}+\delta$ and $\delta>0$ is sufficiently small. Therefore the Dirichlet problem

$$
\begin{aligned}
& A v=f \text { in } B\left(R_{1}, R_{2}\right), \\
& \gamma^{1} v=g, \\
& \gamma^{2} v=0,
\end{aligned}
$$

with $\Gamma_{j}=\left\{x|| x \mid=R_{j}\right\}$ has a unique solution $v \in H^{2 m}\left(B\left(R_{1}, R_{2}\right)\right)$ such that $\left(B=B\left(R_{1}, R_{2}\right)\right)$

$$
\|v\|_{2 m, B} \leqq c\left(\|f\|_{0, B}+\|g\|\left(X_{1}\right)\right) .
$$

We choose a smoothing function $\varphi \in \mathscr{D}\left(\mathbf{R}^{\prime \prime}\right)$ such that

$$
\varphi(x)=\left\{\begin{array}{l}
1,|x| \leqq R_{1}+\delta / 3 \\
0,|x| \geqq R_{1}+2 \delta / 3
\end{array}\right.
$$

and define $\tilde{v} \in H_{\mathrm{vox}}^{2 m}\left(\bar{\Omega}_{1}\right)$ as the zero continuation of $v \varphi$ outside $B\left(R_{1}, R_{2}\right)$. We have

$$
\|\tilde{v}\|_{2 m, \Omega_{1}} \leqq c\left(\|f\|_{0, B}+\|g\|\left(X_{1}\right)\right) .
$$

Furthermore, let $w \in H_{0 w}^{m}\left(\Omega_{1}\right)$ be a solution of the problem

$$
B(w, \varphi)=(f \mid \varphi)_{0, \Omega_{1}}-B(\tilde{v}, \varphi), \quad \varphi \in H_{0 w}^{m}\left(\Omega_{1}\right) .
$$

It holds that $w \in H_{\text {loc }}^{2 m}(\bar{\Omega})$ with

$$
\begin{aligned}
& A w=f-A \tilde{v}, \\
& \gamma^{1} w=0 .
\end{aligned}
$$

Thus by defining $u=w+\tilde{v}$ we have found a function $u \in J\left(\Omega_{1}\right), \mathscr{P}_{1} u=(f ; g)$. Accordingly we have $R\left(\mathscr{P}_{1}\right)=K\left(\Omega_{1}\right) \times X_{1}$.

By regularity results for elliptic equations we conclude

$$
\|u\|_{2 m, \Omega_{1}(R)} \leqq c(R)\left(\left\|J^{1}\right\| K\left(\Omega_{1}\right)+\|g\|\left(X_{1}\right)+\|u\|_{0, \Omega_{1}(R+1)}\right) .
$$

On the other hand (4.44) implies

$$
\||| w \mid\|_{m, p, \Omega_{1}} \leqq c\left(\|f\|\left(K\left(\Omega_{1}\right)+\|\tilde{v}\|_{m, p, \Omega_{1}}\right)\right) .
$$

Relations (4.46), (4.47) and (4.43) yield

$$
\left\|\mathscr{P}_{1}^{-1}(f ; g)\right\|_{2 m, \Omega_{1}(R)} \leqq c(R)\left(\|f\|\left(K\left(\Omega_{1}\right)+\|g\|\left(X_{1}\right)\right),\right.
$$

as required for (A6). The proof for $\left(\mathscr{P}_{1}^{*}\right)^{-1}$ is same. 
Let us formulate our main result of this section. By Lemma 4.4 we conclude from Theorem 3.6

Theorem 4.5. Let $A$ be a strongly elliptic operator given by (4.1) in a smooth exterior domain $\Omega$. Furthermore, let assumptions (B1) and (B2) be satisfied and let the boundary operators $\left\{B_{j}\right\}_{j=0}^{m-1}$ be given such that $\left(A ;\left\{B_{j}\right\}_{j=0}^{m-1}\right)$ is a regular problem. If the spaces $K(\Omega), J(\Omega)$ and $J^{*}(\Omega)$ are defined by (4.30)-(4.32), then the operator $\mathscr{P} u=\left(A u ; B_{0} u, \ldots, B_{m-1} u\right), \mathscr{P}: J(\Omega) \rightarrow K(\Omega) \times X_{0}$ is an indexed operator with the index $\varkappa=\varkappa_{2}$, where $\varkappa_{2}$ is the index of the operator $\mathscr{P}_{2}$ (Section 3 ) referring to a boundary problem for a bounded domain. For the range $R(\mathscr{P})$ holds the characterization $(f ; g) \in R(\mathscr{P})$ if and only if $(f ; g) \in K(\Omega) \times X_{0}$ such that

for all $v \in N\left(\mathscr{P}^{*}\right)$.

$$
(f \mid v)_{0, \Omega}-\sum_{j=0}^{m-1}\left\langle g_{j} \mid T_{j} u\right\rangle_{0, \Gamma_{0}}=0
$$

Remark 4.6. The assumption (4.38) can be replaced e.g. by

$$
\left|a_{\alpha \beta}(x)\right| \leqq c \varrho(x) p_{m,|\alpha|}(x) p_{m,|\beta|}(x)
$$

for all $|\alpha|+|\beta|<2 m$, where $\varrho$ tends to zero at infinity.

\section{Radiation problems}

Here we consider problems which describe the radiation of the time-harmonic waves physically. The typical second order example is the Helmholtz equation $(k>0)$

$$
\begin{aligned}
& \left(\Delta+k^{2}\right) u=f, \\
& \left.B u\right|_{\Gamma_{0}}=g
\end{aligned}
$$

with the Sommerfeld type radiation condition

$$
\frac{\partial}{\partial r} u-i k u \in L^{2}(\Omega)
$$

By condition (5.2) the wave is required to be outgoing. The incoming wave can be fixed if we, instead of (5.2), employ the condition

$$
\frac{\partial}{\partial r} u+i k u \in L^{2}(\Omega)
$$

The theory of the exterior problem (5.1) and (5.2) (or (5.3)) is well-studied. The first arguments showing the uniqueness of solutions with Dirichlet or Neumann boundary conditions were based on Rellich's growth estimate, Rellich [29].

$$
\liminf _{R \rightarrow \infty} R^{-1} \int_{R_{0} \leqq|x| \leqq R}|u|^{2} d x>0
$$


for solutions $u \neq 0$ of the equation $\left(\Delta+k^{2}\right) u=0,|x| \geqq R_{0}$. In the case of the more general second order equation

$$
\sum_{i, j=1}^{n} \partial_{i}\left(a_{i j} \partial_{j} u\right)+\sum_{j=1}^{n} a_{j} \partial_{j} u+\left(k^{2}+a_{0}\right) u=f
$$

Rellich-type growth estimates were proved by Jäger [13] and Kato [15]. For extensions of Rellich's results for higher order operators see Agmon and Hörmander [2] and Hörmander [12].

Existence results were first achieved by Eirdus [7], where the principle of the limiting absorption for exterior radiation problems was introduced. These existence results were later improved by Jäger [13] and by Saito [30].

The articles mentioned above deal with the Dirichlet- or the Neumann type boundary condition or the whole space problem. Other boundary conditions have been studied by Levine [20], where a uniqueness result which also covers the third boundary value problem was proved. Furthermore, Danilova [5] treated an oblique problem for the damped Helmholtz equation. Finally, Witsch [43] proved a Fredholm theorem for general non-tangential second order oblique problems.

The case of the higher order equations, which we, differently from (1.2), write here as $(\lambda>0)$

with

$$
\begin{aligned}
& (A-\lambda) u=f, \\
& \left.B_{j} u\right|_{\Gamma_{0}}=g_{j}, \quad j=0, \ldots, m-1,
\end{aligned}
$$

$$
A u=\sum_{0 \leqq|\alpha|,|\beta| \leqq m}(-1)^{|\alpha|} \partial^{\alpha}\left(a_{\alpha \beta} \partial^{\beta} u\right),
$$

has been elaborated by Eĭdus [8], Finoženok [9], Grušin [10] and Vaŭnberg [35], [36] as well as by Vogelsang [38], [39]. In particular, Vaŭnberg derives in [36] a Fredholm type theorem for general radiation problems with regular boundary conditions. However, the orthogonality conditions were not described. In this section we shall see that Theorem 3.6 also applies to radiation problems of the order $2 m$ and yields a Fredholm result with explicit solvability conditions. By assumptions (A1)-(A6) we presuppose some knowledge of the auxiliary Dirichlet problem. Thus, to employ Theorem 3.6 for a great class of elliptic radiation problems, we use the results of Vogelsang [38], [39].

We have to recall some notations and assumptions of [39]. For every positive parameter $\delta$ we use the weight functions

$$
\begin{gathered}
q(x)=(1+|x|)^{-\delta} \\
p(x)=(1+|x|)^{-1 / 2-\delta}, \\
\varrho(x)=(1+|x|)^{1-\delta} .
\end{gathered}
$$

We define

$$
C_{*}^{k}(\bar{\Omega})=\left\{u \in C^{k}(\bar{\Omega})\left|\sup _{|\alpha| \leqq k, x \in \Omega}\right| \partial^{\alpha} u(x) \mid<\infty\right\}
$$


For any weight function $t(x)>0$ we employ the norm

and we abbreviate

$$
\|u\|_{k, t, \Omega}=\left(\sum_{|\alpha| \leqq k}\left\|t \partial^{\alpha} u\right\|_{0, \Omega}^{2}\right)^{1 / 2}
$$

$$
C_{* *}^{k}(\bar{\Omega})=\left\{u \in C_{*}^{k}(\bar{\Omega}) \mid\|u\|_{k, t, \Omega}<\infty\right\} .
$$

The space $H_{t}^{k}(\Omega)$ is defined as the closure of $C_{* *}^{k}(\bar{\Omega})$ with respect to the norm $\|\cdot\|_{k, t, \Omega}$ and the space $H_{0 t}^{k}(\Omega)$ describing the homogeneous Dirichlet boundary conditions is the closure of $\mathscr{D}(\Omega)$ in $H_{t}^{k}(\Omega)$.

The following assumptions shall be employed:

(CI1) The operator $A$ obeys $a_{\alpha \beta} \in C^{\infty}(\bar{\Omega}), a_{\alpha \beta}=a_{\alpha \beta} \in \mathbf{R}$ and $A$ is uniformly strongly elliptic:

$$
\sum_{|\alpha|=|\beta|=m} a_{\alpha \beta}(x) \xi^{x+\beta} \geqq a_{0}|\xi|^{2 m}, \quad(x, \xi) \in \bar{\Omega} \times \mathbf{R}^{n}
$$

for a constant $a_{0}>0$.

(CI2) There exist constants $a_{\alpha \beta}^{*} \in \mathbf{R}$ such that $a_{\alpha \beta}^{*}=a_{\beta \alpha}^{*}, a_{00}^{*}=0$ and

$$
\left|a_{\alpha \beta}(x)-a_{\alpha \beta}^{*}\right| \leqq c|x|^{-1}, \quad\left|\partial^{\tau} a_{\alpha \beta}(x)\right| \leqq c|x|^{-2}, \quad|\tau| \geqq 1 .
$$

We use the polynomial

$$
P^{*}(\xi)=\sum_{|\alpha|,|\beta| \leqq m} a_{\alpha \beta}^{*} i^{|\beta|-|\alpha|} \xi^{\alpha+\beta}, \quad \xi \in \mathbf{R}^{n} .
$$

Let $\mathscr{N}=\mathscr{N}(\lambda)$ be the surface of the real zeros

$$
\mathscr{N}=\left\{\xi \in \mathbf{R}^{n} \mid P^{*}(\xi)-\lambda=0\right\} .
$$

(CI3) We assume that $\mathscr{N}$ is connected and that for any $\eta \in \mathcal{N}$

$$
D_{\eta} P^{*}(\eta) \neq 0,\left(D_{\eta_{i} \eta_{j}} P^{*}(\eta)\right)_{i, j=1}^{n} \text { is positive definite. }
$$

Furthermore, let us write

$$
\Phi(\eta, \xi)=P^{*}(\eta+\xi)-D_{\eta} P^{*}(\eta) \xi-P^{*}(\eta),(\eta, \xi) \in \mathscr{N} \times \mathbf{R}^{n} .
$$

We assume that the requirement of a stronger ellipticity

(CI4) $\quad \Phi(\eta, \xi) \geqq c\left(|\xi|^{2}+|\xi|^{2 m}\right)$

$$
|\xi| D_{|\eta|} \Phi(\xi, \eta)-\Phi(\eta, \xi) \geqq c\left(|\xi|^{2}+|\xi|^{2 m}\right),(\eta, \xi) \in \mathscr{N} \times \mathbf{R}^{n}
$$

is valid. Here $D_{\xi}=\nabla_{\xi}, D_{|\xi|}=|\xi|^{-1}\left(\xi \cdot \nabla_{\xi}\right)$.

The assumptions (CI1) - (CI4) are essentially those of [39]. However, we employ our general assumption on the smoothness of the coefficients. The condition that the coefficients are real does not appear in [39].

We remark that by $a_{\alpha \beta}^{*}=a_{\beta \alpha}^{*}$ we may without any loss of generality suppose that $a_{\alpha \beta}^{*}=0$ if $|\alpha|+|\beta|$ is odd. 
The radiation problem is defined by using the notion of the characteristic function $s \in C^{\infty}\left(\mathbf{R}^{n} \backslash\{0\}\right)$ (cf. Hörmander [11], Schulenberger-Wilcox [34]) given by

$$
s(x)=\sigma(\hat{x}) \cdot x, \quad \hat{x}=|x|^{-1} x,
$$

where $\sigma: S^{n-1} \rightarrow \mathscr{N}(\lambda), S^{n-1}=\left\{x \in \mathbf{R}^{n}|| x \mid=1\right\}$, is the inverse of the Gauss mapping satisfying $x \cdot \sigma(\hat{x})>0$ and

$$
\hat{x}=\varkappa(\hat{x}) D_{\xi} P^{*}(\sigma(\hat{x}))
$$

where $x(\hat{x})>0$.

The radiation condition now appears in the form

$$
\partial^{\alpha}\left(e^{-i s} u\right) \in L_{q}^{2}\left(E\left(R_{0}\right)\right), \quad 1 \leqq|\alpha| \leqq m,
$$

with $E\left(R_{0}\right)=\left\{x \in \mathbf{R}^{n}|| x \mid>R_{0}\right\}$, where $R_{0}>0$ is any number such that $E\left(R_{0}\right) \subset \Omega$. The condition (5.22) is "outgoing". The "incoming" condition reads

$$
\partial^{\alpha}\left(e^{i s} u\right) \in L_{q}^{2}\left(E\left(R_{0}\right)\right), \quad 1 \leqq|\alpha| \leqq m .
$$

We define the spaces $J(\Omega), J^{*}(\Omega)$ and $K(\Omega)$ by setting

$$
\begin{gathered}
K(\Omega)=L_{\varrho}^{2}(\Omega), \\
J(\Omega)=\left\{u \in H_{p}^{2 m}(\Omega)\left|\partial^{\alpha}\left(e^{-i s} u\right) \in L_{q}^{2}\left(E\left(R_{0}\right)\right), \quad 1 \leqq\right| \alpha \mid \leqq m, \quad(A-i) u \in K(\Omega)\right\}, \\
J^{*}(\Omega)=\left\{u \in H_{p}^{2 m}(\Omega)\left|\partial^{\alpha}\left(e^{i s} u\right) \in L_{q}^{2}\left(E\left(R_{0}\right)\right), \quad 1 \leqq\right| \alpha \mid \leqq m, \quad(A-i) u \in K(\Omega)\right\} .
\end{gathered}
$$

Now, the operators $\mathscr{P}: J(\Omega) \rightarrow K(\Omega) \times X_{0}$ and $\mathscr{P}^{*}: J^{*}(\Omega) \rightarrow K(\Omega) \times X_{0}$ are given by $\left(A^{*}=A\right)$

$$
\begin{aligned}
\mathscr{P} u & =\left(A u-\lambda u ; B_{0} u, \ldots, B_{m-1} u\right), \\
\mathscr{P}^{*} u & =\left(A u-\lambda u ; C_{0} u, \ldots, C_{m-1} u\right) .
\end{aligned}
$$

We shall apply Theorem 3.6 in the case where for the Dirichlet problem the following spectral result is valid.

(CII) For the exterior domain $\Omega_{1} \subset \Omega$ the problem

$$
\begin{aligned}
& (A-\lambda) u=0, \quad u \in J\left(\Omega_{1}\right), \\
& \left.\gamma_{j} u\right|_{\Gamma_{1}}=0, \quad j=0, \ldots, m-1,
\end{aligned}
$$

has only the trivial solution $u=0$.

We refer to Vogelsang [38] for a discussion of the cases where this assumption is valid. Note that since the coefficients of $A$ are real, it holds that the condition $u \in J\left(\Omega_{1}\right)$ is equivalent to $\bar{u} \in J^{*}\left(\Omega_{1}\right)$. Therefore, if (CII) is valid, then the adjoint 
problem

$$
\begin{aligned}
& (A-\lambda) u=0, \quad u \in J^{*}\left(\Omega_{1}\right), \\
& \left.\gamma_{j} u\right|_{\Gamma_{1}}=0, \quad j=0, \ldots, m-1,
\end{aligned}
$$

only has the trivial solution.

Furthermore note that for $u \in H_{p}^{m}\left(\Omega_{1}\right)$ the condition $\left.\gamma_{j} u\right|_{\Gamma_{1}}=0, j=0, \ldots, m-1$ is equivalent to the requirement $u \in H_{0 p}^{m}\left(\Omega_{1}\right)$ and thus the results of [38] can be adapted.

We are able to state

Lemma 5.1. Let the conditions (CI) and (CII) be satisfied. If for the parameter $\delta$ holds $0<\delta \leqq 1 / 4$, then the assumptions (A1)-(A3), (A5) and (A6) are valid.

Proof. The validity of (A1) and (A2) is a direct consequence of the definitions (5.24)-(5.26). For (A3) we note that if $u \in J^{(*)}(\Omega)$ and $f \in K(\Omega), 0<\delta \leqq 1 / 4$, then we have

$$
\int_{\Omega}|u \bar{f}| d x \leqq \int_{\Omega}(1+|x|)^{-1 / 2-\delta}|u|(1+|x|)^{1-\delta}|f| d x \leqq\|u\|_{0, p, \Omega}\|f\|_{0, \varrho, \Omega}<\infty .
$$

The condition (CII) is essentially used to guarantee that $N\left(\mathscr{P}_{1}\right)=N\left(\mathscr{P}_{1}^{*}\right)=\{0\}$ and that $R\left(\mathscr{P}_{1}\right)=R\left(\mathscr{P}_{1}^{*}\right)=K\left(\Omega_{1}\right) \times X_{1}$. The first of these assertions follows by [39], Satz 1. The latter is a consequence of [39], Satz 4. Furthermore, we obtain by the same result (cf. proof of Lemma 4.4)

$$
\sum_{1 \leqq|\alpha| \leqq m}\left\|\partial^{\alpha}\left(e^{-i s} u\right)\right\|_{0, q, E\left(R_{0}\right)}+\|u\|_{2 m, p, \Omega_{1}} \leqq c\left(R_{0}\right)\left(\|f\|\left(K\left(\Omega_{1}\right)\right)+\|g\|\left(X_{1}\right)\right)
$$

for $\mathscr{P}_{1} u=(f ; g)$ and

$$
\sum_{1 \leqq|\alpha| \leqq m}\left\|\partial^{\alpha}\left(e^{i s} u\right)\right\|_{0, q, E\left(R_{0}\right)}+\|u\|_{2 m, p, \Omega_{1}} \leqq c\left(R_{0}\right)\left(\|f\|\left(K\left(\Omega_{1}\right)\right)+\|g\|\left(X_{1}\right)\right)
$$

for $\mathscr{P}_{1}^{*} u=(f ; g)$.

These estimates imply that (A5) and (A6) are valid.

The verification of (A4) requires the following:

Lemma 5.2. If $u \in J(\Omega), v \in J^{*}(\Omega)$ are given such that they vanish in a neighbourhood of the boundary $\partial \Omega$, then we have

$$
((A-\lambda) u \mid v)_{0, \Omega}=(u \mid(A-\lambda) v)_{0, \Omega} .
$$

Proof. Choose $\psi \in \mathscr{D}(\mathbf{R}), \psi \geqq 0$, such that $\psi(t)=1, t \in[0,1]$ and define

$$
\psi_{R}(x)=\psi\left(R^{-1} s(x)\right), R>0 .
$$

Then we have by Green's formula

$$
\begin{aligned}
& ((A-\lambda) u \mid v)_{0, \Omega}-(u \mid(A-\lambda) v)_{0, \Omega}=\lim _{R \rightarrow \infty}\left\{\left(A u \mid \psi_{R} v\right)_{0, \Omega}-\left(\psi_{R} u \mid A v\right)_{0, \Omega}\right\} \\
= & \lim _{R \rightarrow \infty} \sum_{|\alpha|,|\beta| \leqq m}\left\{\left(a_{\alpha \beta} \partial^{\beta} u \mid \partial^{\alpha}\left(\psi_{R} v\right)\right)_{0, \Omega}-\left(a_{\alpha \beta} \partial^{\alpha}\left(\psi_{R} u\right) \mid \partial^{\beta} v\right)_{0, \Omega}\right\}=: \lim _{R \rightarrow \infty} I_{R} .
\end{aligned}
$$

We apply Leibniz's rule to $\partial^{\alpha}\left(\psi_{R} v\right), \partial^{\alpha}\left(\psi_{R} u\right)$, and split the resulting sum into 
terms, where $\psi_{R}$ is differentiated zero, one or more than one time. This yields

$$
\begin{aligned}
& I_{R}=\sum_{|\alpha|,|\beta| \leqq m}\left\{\left(a_{\alpha \beta} \psi_{R} \partial^{\beta} u \mid \partial^{\alpha} v\right)_{0, \Omega}-\left(a_{\alpha \beta} \psi_{R} \partial^{\alpha} u \mid \partial^{\beta} v\right)_{0, \Omega}\right\}
\end{aligned}
$$

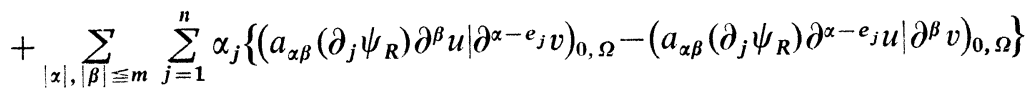

$$
\begin{aligned}
& +\sum_{|\alpha|,|\beta| \leqq m} \sum_{\substack{\gamma \equiv \alpha \\
|\gamma| \geqq 2}}\left(\begin{array}{l}
\alpha \\
\gamma
\end{array}\right)\left\{\left(a_{\alpha \beta}\left(\partial^{\gamma} \psi_{R}\right) \partial^{\beta} u \mid \partial^{\alpha-\gamma} v\right)_{0, \Omega}-\left(a_{\alpha \beta}\left(\partial^{\gamma} \psi_{R}\right) \partial^{\alpha-\gamma} u \mid \partial^{\beta} v\right)_{0, \Omega}\right\} .
\end{aligned}
$$

Since $a_{\alpha \beta}=a_{\beta \alpha}$, the first sum vanishes identically. For the derivatives of $\psi_{R}$

$$
\left|\left(\partial^{\gamma} \psi_{R}\right)(x)\right| \leqq c R^{-|\gamma|} \leqq c|x|^{-|\gamma|},
$$

where $c$ is a constant independent of $R$. For (5.35) we have used the property

$$
\left|\left(\partial^{\tau} s\right)(x)\right| \leqq c|x|^{-|\tau|+1}
$$

of the characteristic function.

Let $\sigma_{1}=\max \left\{\sigma(\hat{x}) \cdot \hat{x} \mid x \in S^{n-1}\right\}$. Then the function $\psi_{R}$ is identically one in the ball $B\left(\sigma_{1}^{-1} R\right)=\left\{x|| x \mid<\sigma_{1}^{-1} R\right\}$. Thus, by (5.35), any term in the third sum in (5.34) can be estimated by

$$
c \sum_{|\alpha|,|\beta| \leqq m} \int_{|x|>\sigma_{1}^{-1} R}|x|^{-2}\left|\partial^{\beta} u\right|\left|\partial^{\alpha} v\right| d x .
$$

Since $\left|\partial^{\beta} u\right|,\left|\partial^{\alpha} v\right|$ belong to $L_{p}^{2}(\Omega)$, the integrals in (5.37) tend to zero as $R$ tends to infinity.

It remains to show that the second term in (5.34) tends to zero. For this note that

$$
\partial_{j} \psi_{R}(x)=\left(\partial_{j} s\right) \tilde{\psi}_{R},
$$

where

$$
\left|\tilde{\psi}_{R}(x)\right| \leqq\left|R^{-1} \psi^{\prime}\left(R^{-1} s(x)\right)\right| \leqq c|x|^{-1} .
$$

Furthermore, if $\alpha_{j} \neq 0$, we get by Leibniz's rule $\left(e_{j}=\left(\delta_{k j}\right)_{k=1}^{n}\right)$

$$
\begin{aligned}
& \partial^{\alpha} u=\partial^{\alpha-e_{j}} \partial_{j}\left(e^{i s}\left(e^{-i s} u\right)\right)=\partial^{\alpha-e_{j}}\left(i\left(\partial_{j} s\right) u+e^{i s} \partial_{j}\left(e^{-i s} u\right)\right) \\
& =i\left(\partial_{j} s\right) \partial^{\alpha-e_{j}} u+\left[i \sum_{0<\mu \leqq \alpha-e_{j}}\left(\begin{array}{c}
\alpha-e_{j} \\
\mu
\end{array}\right)\left(\partial^{\mu+e_{j} s}\right)\left(\partial^{\alpha-e_{j}-\mu} u\right)\right. \\
& \left.+\sum_{0 \leqq \mu \leqq \alpha-e_{j}}\left(\begin{array}{c}
\alpha-e_{j} \\
\mu
\end{array}\right)\left(\partial^{\mu} e^{i s}\right) \partial^{\alpha-\mu}\left(e^{-i s} u\right)\right]=i\left(\partial_{j} s\right) \partial^{\alpha-e_{j} u+i u_{\alpha, j}},
\end{aligned}
$$

where the term in the brackets has been abbreviated by $i u_{\alpha, j}$. By means of (5.36) we see from the definition of $J(\Omega)$ that $u_{\alpha, j} \in L_{q}^{2}\left(E\left(R_{0}\right)\right)$. In the same way one realizes that

$$
\partial^{\alpha} v=-i\left(\partial_{j} s\right) \partial^{\alpha-e_{j}} v+i v_{\alpha, j}, v_{\alpha, j} \in L_{q}^{2}\left(E\left(R_{0}\right)\right)
$$


Hence, we get from (5.38), (5.40) and (5.41)

$$
\begin{gathered}
\left(\partial_{j} \psi_{R}\right) \partial^{\alpha-e_{j} v}=i \tilde{\psi}_{R} \partial^{\alpha} v+\tilde{\psi}_{R} v_{\alpha j}, \\
\left(\partial_{j} \psi_{R}\right) \partial^{\alpha-e_{j} v}=-i \tilde{\psi}_{R} \partial^{\alpha} u-\tilde{\psi}_{R} u_{\alpha, j}
\end{gathered}
$$

Insertion of these relations into the second term in (5.34) yields

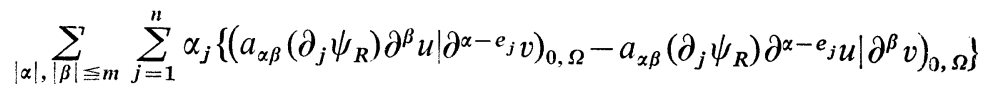

$$
\begin{aligned}
& =\sum_{|\alpha|,|\beta| \leqq m} \sum_{j=1}^{n} \alpha_{j}\left\{\left(-i\left(a_{\alpha \beta} \tilde{\psi}_{R} \partial^{\beta} u \mid \partial^{\alpha} v\right)_{0, \Omega}+i\left(a_{\alpha \beta} \tilde{\psi}_{R} \partial^{\alpha} u \mid \partial^{\beta} v\right)_{0, \Omega}\right\}\right. \\
& +\sum_{|\alpha|,|\beta| \leqq m} \sum_{j=1}^{n} \alpha_{j}\left\{\left(a_{\alpha \beta} \tilde{\psi}_{R} \partial^{\beta} u \mid v_{\alpha, j}\right)_{0, \Omega}+\left(a_{\alpha \beta} \tilde{\psi}_{R} u_{\alpha, j} \mid \partial^{\beta} v\right)_{0, \Omega}\right\}
\end{aligned}
$$

Since $0<\delta \leqq 1 / 4$, the last sum tends to zero as $R$ tends to infinity. We split the first sum on the right side of (5.42) into

where

$$
S=S_{1}+S_{2},
$$

$$
\begin{aligned}
& S_{1}=i \sum_{|\alpha|,|\beta| \leqq m} \sum_{j=1}^{n} \alpha_{j}\left(\left(a_{\alpha \beta}^{*}-a_{\alpha \beta}\right) \tilde{\psi}_{R} \partial^{\beta} u \mid \partial^{\alpha} v\right)_{0, \Omega}-\left(\left(a_{\alpha \beta}^{*}-a_{\alpha \beta}\right) \tilde{\psi}_{R} \partial^{\alpha} u \mid \partial^{\beta} v\right) \\
& S_{2}=i \sum_{|\alpha|,|\beta| \leqq m} \sum_{j=1}^{n} \alpha_{j}\left(\left(a_{\alpha \beta}^{*} \tilde{\psi}_{R} \partial^{\alpha} u \mid \partial^{\beta} v\right)_{0, \Omega}-\left(a_{\alpha \beta}^{*} \tilde{\psi}_{R} \partial^{\beta} u \mid \partial^{\alpha} v\right)_{0, \Omega}\right) .
\end{aligned}
$$

The term $S_{1}$ can be estimated as in (5.37) and tends to zero. Since $a_{\alpha \beta}^{*}=a_{\beta \alpha}^{*}, S_{2}$ can be written in the form

where

$$
S_{2}=\sum_{|\alpha|,|\beta| \leqq m}\left(b_{\alpha \beta} \tilde{\psi}_{R} \partial^{\alpha} u \mid \partial^{\beta} v\right)_{0, \Omega},
$$

$$
b_{\alpha \beta}=\sum_{j=1}^{n} i\left(\alpha_{j}-\beta_{j}\right) a_{\alpha \beta}^{*}=\bar{b}_{\beta \alpha}=-b_{\alpha \beta} .
$$

Since $\tilde{\psi}_{R}$ has compact support, integration by parts and application of Leibniz's rule gives

$$
\begin{gathered}
S_{2}=\sum_{|\alpha|,|\beta| \leqq m}\left(\tilde{\psi}_{R} b_{\alpha \beta}(-1)^{|\beta|} \partial^{\beta+\alpha} u \mid v\right)_{0, \Omega} \\
+\sum_{|\alpha|,|\beta| \leqq m} \sum_{0<\gamma \leqq \beta}\left(\begin{array}{l}
\beta \\
\gamma
\end{array}\right)\left(b_{\alpha \beta} \partial^{\beta-\gamma+\alpha} u \mid\left(\partial^{\gamma} \tilde{\psi}_{R}\right) v\right)_{0, \Omega} .
\end{gathered}
$$

In the second sum the derivatives $\partial^{\beta-\gamma}$ can be carried to the right side again, yielding integrals which can be estimated by (5.37) and hence tend to zero.

Since $a_{\alpha \beta}^{*}=0$ if $|\alpha|+|\beta|$ is odd, we have $(-1)^{|\alpha|+|\beta|}=1$ or $b_{\alpha \beta}=0$. This 
gives the formulae

$$
\begin{aligned}
& \sum_{|\alpha|,|\beta| \leqq m}\left(\tilde{\psi}_{R} b_{\alpha \beta}(-1)^{|\beta|} \partial^{\beta+\alpha} u \mid v\right)_{0, \Omega}=\sum_{|\alpha|,|\beta| \leqq m}\left(\tilde{\psi}_{R} b_{\alpha \beta} \partial^{\beta} u \mid \partial^{\alpha} v\right)_{0, \Omega}+o(1), \\
& \sum_{|\alpha|,|\beta| \leqq m}\left(\tilde{\psi}_{R} b_{\alpha \beta}(-1)^{|\beta|} \partial^{\beta+\alpha} u \mid v\right)_{0, \Omega}=\sum_{|\alpha|,|\beta| \leqq m}\left(\tilde{\psi}_{R} b_{\alpha \beta} \partial^{\alpha} u \mid \partial^{\beta} v\right)_{0, \Omega}+o(1),
\end{aligned}
$$

where $o(1)$ denotes an expression similar to the last sum in (5.43). Combining (5.43)-(5.45) we obtain $S_{2}=o(1)$. Thus the lemma is proved.

By Lemma 5.1 and Lemma 5.2 we conclude from Theorem 3.6.

Theorem 5.3. Let $A$ be a uniformly strongly elliptic operator in the smooth exterior domain $\Omega$ such that the assumptions (CI) and (CII) are satisfied. Then the regular exterior boundary value problem $(\lambda>0,0<\delta \leqq 1 / 4)$

$$
\begin{aligned}
& (A-\lambda) u=f, \quad u \in H_{p}^{2 m}(\Omega), \quad f \in L_{\varrho}^{2}(\Omega), \\
& \left.B_{j} u\right|_{\Gamma_{0}}=g_{j} \in H^{2 m-m_{j}-1 / 2}\left(\Gamma_{0}\right), \quad j=0,1, \ldots, m-1, \\
& \partial^{\alpha}\left(e^{-i s} u\right) \in L_{q}^{2}\left(E\left(R_{0}\right)\right), \quad 1 \leqq|\alpha| \leqq m,
\end{aligned}
$$

has a finite index $\chi=\chi_{2}$ (for notation see Section 3 ).

The solvability conditions for (5.46) read: for $(f ; g) \in L_{\varrho}^{2}(\Omega) \times \prod_{j=0}^{m-1} H^{2 m-m_{j}-1 / 2}\left(\Gamma_{0}\right)$ there exists a solution if and only if

$$
(f \mid v)_{0, \Omega}-\sum_{j=0}^{m-1}\left\langle g_{j} \mid T_{j} v\right\rangle_{0, \Gamma_{0}}=0,
$$

holds for all $v$ which are solutions of the homogeneous adjoint problem

$$
\begin{aligned}
& (A-\lambda) v=0, \quad v \in H_{p}^{2 m}(\Omega), \\
& \left.C_{j} v\right|_{\Gamma_{0}}=0, \quad j=0,1, \ldots, m-1, \\
& \partial^{\alpha}\left(e^{i s} v\right) \in L_{q}^{2}\left(E\left(R_{0}\right)\right), \quad 1 \leqq|\alpha| \leqq m .
\end{aligned}
$$

\section{Polynomials of the Laplacian}

As a final example we consider differential operators $A$ of the type

$$
A=P(L),
$$

where $L$ is a uniformly strongly elliptic partial differential operator of second order

$$
L u(x)=\sum_{i, j=1}^{n} \partial_{i}\left(a_{i j}(x) \partial_{j} u(x)\right)+a(x) u(x),
$$

where $a_{i j}(x), a(x) \in \mathbf{R}$ and $a_{i j}=a_{j i}$ and where $P=P(t)$ is a normalized polynomial with real coefficients of degree $m$ in one variable. Hence $A^{*}=A$. Since we shall base our discussion of problem (2.15) on the results in [45], let us briefly recall the assumptions on $L$ and $P$ and the results of this paper. 
For the coefficients of $L$ we require

$$
a_{i j}-\delta_{i j}, \quad a \in \mathscr{S}\left(\mathbf{R}^{n}\right),
$$

where $\delta_{i j}$ is Kronecker's symbol, and where $\mathscr{S}(\Omega)$ denotes Schwartz's space

$$
\mathscr{S}(\Omega)=\left\{\left.\varphi \in C^{\infty}(\Omega)\left|\lim _{|x| \rightarrow \infty}\right| x\right|^{k} \varphi(x)=0 \text { for any } k \in \mathbf{N}\right\}
$$

of rapidly vanishing smooth functions.

The polynomial $P$ does neither vanish at $t=0$ (cf. [32] to avoid this assumption) nor at any $t$ such that $(L-t) u=0$ has a nontrivial solution in $L^{2}\left(\mathbf{R}^{n}\right)$. The latter seems not to be a severe restriction for one can change the coefficients in some bounded region to avoid this assumption. The zeros of $P$ are denoted by $-k_{\varrho}^{2}$, $\varrho=1, \ldots, q$, where $\operatorname{Im} k_{\varrho} \geqq 0, k_{\varrho} \neq \leq 0, k_{\varrho}^{2} \neq k_{\tau}^{2}$ for $\varrho \neq \tau$. By $r_{\varrho}$ we mean the order of $-k_{\varrho}^{2}$ as a zero of $P$. Hence

$$
P(t)=\prod_{\varrho=1}^{q}\left(t+k_{\varrho}^{2}\right)^{r_{\varrho}} .
$$

The results of $[45, \mathrm{Ch} .2]$ are collected in the following lemma. For its formulation we denote by $\Omega$ some exterior domain, by $\Lambda$ the first order operator

and by $\hat{\mathscr{S}}(\Omega)$ the space

$$
\Lambda=\sum_{i=1}^{n} x_{i} \partial_{i}
$$

$$
\hat{\mathscr{S}}(\Omega)=\left\{\left.\varphi \in C^{\infty}(\Omega)\left|\lim _{|x| \rightarrow \infty}\right| x\right|^{-k} \varphi(x)=0 \text { for some } k \in \mathbf{N}\right\} .
$$

We have

Lemma 6.1. Any solution $u \in \hat{\mathscr{S}}(\Omega)$ of the equation

can be decomposed as

$$
P(L) u=f \in \mathscr{S}(\Omega)
$$

$$
u=\sum_{\mu=1}^{q} \sum_{v=0}^{r_{\mu}-1} \Lambda^{v} u_{\mu, v}
$$

where $u_{\mu, v}\left(\mu=1, \ldots, q ; v=0, \ldots, r_{\mu-1}\right)$ belong to $\hat{\mathscr{S}}(\Omega)$ and solve

$$
\left(L+k_{\mu}^{2}\right) u_{\mu, v}=f_{\mu, v} \in \mathscr{S}(\Omega) .
$$

The functions $u_{\mu, v}$ and $f_{\mu, v}$ can be calculated by application of certain systems of differential operators to the pair $(u, f)$. The decomposition of $u$ is unique in the following sense: If $u_{\mu, v}, v_{\mu, v} \in \hat{\mathscr{S}}(\Omega)$ satisfy

and

$$
\left(L+k_{\mu}^{2}\right) u_{\mu, v} \in \mathscr{S}(\Omega) ;\left(L+k_{\mu}^{2}\right) v_{\mu, v} \in \mathscr{S}(\Omega)
$$

$$
\sum_{\mu=1}^{q} \sum_{v=0}^{r_{\mu-1}} \Lambda^{v} u_{\mu, v}=\sum_{\mu=1}^{q} \sum_{v=0}^{r_{\mu-1}} \Lambda^{v} v_{\mu, v}
$$


then

$$
u_{\mu, v}-v_{\mu, v} \in \mathscr{S}(\Omega) \text {. }
$$

We are interested in solving the problem (1.2) with $A=P(L)$ for $f \in H_{\mathrm{vox}}^{0}(\bar{\Omega})$. Hence we choose

$$
K(\Omega)=H_{\mathrm{vox}}^{0}(\bar{\Omega}) .
$$

Since the components $u_{\mu, v}$ satisfy reduced wave equations, asymptotic conditions for $u$ can be formulated by imposing radiation conditions on $u_{\mu, v}$.

For the definition of $J(\Omega), J^{*}(\Omega)$ we denote by $s=\left(s_{1}, \ldots, s_{q}\right)$ a $q$-vector of real units, $s_{i} \in\{-1,+1\}$ and put $s^{*}=-s$. Then the space $J^{(*)}(\Omega)$ is defined as the space of all functions $u \in H_{\mathrm{loc}}^{2 m}(\bar{\Omega})$ which for some sufficiently large $S>0$ satisfy

$$
\begin{gathered}
\operatorname{supp} P(L) u \subset \Omega(S), \\
\left.u\right|_{\Omega \backslash \bar{\Omega}(S)}=\sum_{\mu=1}^{q} \sum_{v=0}^{r_{\mu-1}} \Lambda^{v} u_{\mu, v},
\end{gathered}
$$

where for the component $u_{\mu, v}$

$$
\begin{gathered}
\left(L+k_{\mu}^{2}\right) u_{\mu, v} \in \mathscr{S}(\Omega \backslash \overline{\Omega(S)}), \\
D u_{\mu, v}-i s_{\mu}^{(*)} k_{\mu} u_{\mu, v} \in L^{2}(\Omega \backslash \overline{\Omega(S)}),
\end{gathered}
$$

with

$$
D:=\sum_{i, j=1}^{n} x_{i}|x|^{-1} a_{i j}(x) \partial_{j} .
$$

Note that the radiation condition $(6.4)^{(*)}$ yields $\mu_{\mu, v} \in H^{2}(\Omega \backslash \overline{\Omega(S)})$ if $\operatorname{Im} k_{\mu}>0$, Jäger [13].

The decomposition of $u$ suggests that we may admit more general conditions at infinity: given $\mu,(6.4)^{(*)}$ gives the same kind of radiation condition for any $v=0, \ldots, r_{\mu}-1$. Only in this case is the validity of Green's identity (2.19) proved in [45, Theorem 3.3].

Lemma 6.2. Let $s \in\{-1,1\}^{q}$ be fixed. Then we have

(i) For any $f \in \mathscr{S}\left(\mathbf{R}^{n}\right)$ there exists a unique solution $u \in \hat{\mathscr{S}}(\mathbf{R})^{n}$ of $P(L) u=f$, satisfying (6.2)-(6.4). There exists a positive integer $l$ and a positive real number $p$ independent of $u$ and $f$, such that for any $R>0$ the solution $u$ can be estimated by

$$
\|u\|_{2 m, B(R)} \leqq c\left\|\left(1+|x|^{2}\right)^{p} f\right\|_{l, \mathbf{R}^{n}},
$$

where $B(R)=\{x|| x \mid<R\}$ and where the constant $c=c(R)$ is independent of $u$ and $f$.

(ii) For any exterior domain $\Omega$, the spaces

respectively

$$
\mathscr{N}(\Omega)=\left\{u \in J(\Omega) \cap H_{0, \mathrm{loc}}^{m}(\Omega) \mid P(L) u=0\right\}
$$

$$
\mathscr{N}^{*}(\Omega)=\left\{u \in J^{*}(\Omega) \cap H_{0,1 \mathrm{loc}}^{m}(\Omega) \mid P(L) u=0\right\}
$$

are of the same finite dimension $d$. 
(iii) For $f \in \mathscr{S}(\bar{\Omega}):=\left\{\left.\hat{f}\right|_{\Omega} \mid \hat{f} \in \mathscr{S}\left(\mathbf{R}^{n}\right)\right\}$ the Dirichlet problem

$$
u \in J(\Omega) \cap H_{0,10 \mathrm{c}}^{m}(\bar{\Omega}), P(L) u=f
$$

is solvable if and only if

$$
(f \mid v)_{0, \Omega}=0, \text { for all } u \in \mathscr{N}^{*}(\Omega) .
$$

Lemma 6.2 does not fit in the framework of the theory established in Chapters 2 and 3. For example (6.6) should be solved for $f \in K(\Omega)$ and also inhomogeneous boundary conditions should be considered. To take this into account we suppose that $\Omega$ has a smooth boundary $\Gamma$ and prove the following estimate:

Lemma 6.3. Let for some $S>\sup \{|x| \mid x \in \Gamma\}$ the function $u \in J(\Omega)$ satisfy

$$
\text { supp } P(L) u \subset \Omega(S) \text {. }
$$

Then for any $R>0$ there exists a constant $c$, depending on $R$ and on $S$, but not on $u$, such that

$$
\|u\|_{2 m, \Omega(R)} \leqq c\left(\|P(L) u\|_{0, \Omega(S)}+\sum_{j=0}^{m-1}\left\|\gamma_{j} u\right\|_{2 m-j-1 / 2, \Gamma}+\|u\|_{0, \Omega(S+1)}\right) .
$$

Proof: Let us consider a testfunction $\chi \in \mathscr{D}\left(\mathbf{R}^{n}\right)$ such that $\chi \equiv 1$ in $B(S+1 / 3)$ and that $\operatorname{supp} \chi \subset \subset B(S+2 / 3)$. Writing

$$
u_{1}=\chi u, \quad u_{2}=(1-\chi) u
$$

and continuing $u_{2}$ by zero, we get from Lemma 6.2 (i)

$$
\begin{gathered}
\left\|u_{2}\right\|_{2 m, \Omega(R)} \\
=\left\|u_{2}\right\|_{2 m, B(R)} \leqq c\left\|\left(1+|x|^{2}\right)^{p} P(L) u_{2}\right\|_{l, \mathrm{R}^{n}} \leqq c\|u\|_{l+2 m-1, B(S+2 / 3) \backslash B(S+1 / 3)}
\end{gathered}
$$

and further from the well-known a priori estimate ([21], p. 149)

$$
\begin{gathered}
\leqq c\left(\left\|P(L) u_{1}\right\|_{0, \Omega(S+1)}+\sum_{j=0}^{m-1}\left\|\gamma_{j} u\right\|_{2 m-j-1 / 2, \Gamma}+\left\|u_{1}\right\|_{0, \Omega(S+1)}\right) \\
\leqq c\left(\|P(L) u\|_{0, \Omega(S)}+\sum_{j=0}^{m-1}\left\|\gamma_{j} u\right\|_{2 m-j-1 / 2, \Gamma}+\|u\|_{0, \Omega(S+1)}+\|u\|_{2 m-1, B(S+2 / 3) \backslash B(S+1 / 3)}\right) .
\end{gathered}
$$$$
\left\|u_{1}\right\|_{2 m, \Omega(R)} \leqq\left\|u_{1}\right\|_{2 m, \Omega(S+1)}
$$

Using interior a priori estimates, $\|u\|_{2 m-1, B(S+2 / 3) \backslash B(S+1 / 3)}$ and $\|u\|_{l+2 m, B(S+2 / 3) \backslash B(S+1 / 3)}$ can be estimated by $\|u\|_{0, B(S+1) \backslash B(S)}$, multiplied by a constant. Now, combining (6.10) and (6.11) we get the assertion.

From Lemma 6.2 and Lemma 6.3 we conclude

Theorem 6.4. For $f \in K(\Omega)$ and $\left(g_{0}, \ldots, g_{m-1}\right) \in H^{2 m-j-1 / 2}(\Gamma)$, there exists a solution $u \in J(\Omega)$ of the problem

$$
\begin{gathered}
P(L) u=f \text { in } \Omega \\
\gamma_{j} u=g_{j}, j=0, \ldots, m-1,
\end{gathered}
$$


if and only if for any $v \in \mathscr{N}^{*}(\Omega)$

$$
(f \mid v)_{0, \Omega}-\sum_{j=0}^{m-1}\left\langle g_{j} \mid T_{j} v\right\rangle_{0, \Gamma}=0 .
$$

Here $\left\{\gamma_{0}, \ldots, \gamma_{m-1}, T_{0}, \ldots, T_{m-1}\right\}$ is a Dirichlet system of order $2 m$ on $\Gamma$ such that Green's formula (2.8) holds with $B_{j}=\gamma_{j}, C_{j}=\gamma_{j}$.

Proof: We first consider the case $g_{j}=0, j=0, \ldots, m-1$. If $f \in K(\Omega)$ satisfies

$$
(f \mid v)_{0, \Omega}=0 \text { for all } v \in \mathscr{N}^{*}(\Omega) \text {, }
$$

we can choose a sequence $f_{n} \in \mathscr{D}(\Omega)$ which tends to $f$ in $L^{2}(\Omega)$ and has the properties

$$
\begin{gathered}
\operatorname{supp} f_{n} \subset \Omega(S), \\
\left(f_{n} \mid v\right)_{0, \Omega}=0 \text { for all } v \in \mathscr{N}^{*}(\Omega),
\end{gathered}
$$

where $S$ is some sufficiently large radius.

By Lemma 6.2 there exists a solution $u_{n} \in J(\Omega)$ of $P(L) u_{n}=f_{n}, \gamma_{j} u_{n}=0$ for any $n \in \mathbf{N}$. Moreover, $u_{n}$ can be chosen in such a way that

$$
\left(u_{n} \mid \psi h\right)_{0, \Omega}=0 \text { for all } h \in \mathscr{N}(\Omega) \text {. }
$$

Here $\psi \in \mathscr{D}(\Omega)$ denotes a fixed function with the property

Under the assumption

$$
\psi h \neq 0, \quad \text { if } \quad h \in \mathcal{N}(\Omega), \quad h \neq 0 .
$$

$$
\sup \left\|u_{n}\right\|_{0, \Omega(s+1)}<\infty,
$$

Lemma 6.3 and Rellich's compactness theorem guarantee the existence of a subsequence $u_{n}^{\prime}$, converging in $L^{2}(\Omega(S+1))$ and hence in $H_{\mathrm{loc}}^{2 m}(\bar{\Omega})$ to an element $u \in H_{\mathrm{loc}}^{2 m}(\bar{\Omega})$. Clearly, $u$ satisfies (6.12) and (6.13) with $g_{j}=0$, as well as

$$
(u \mid \psi h)_{0, \Omega}=0 \text { for all } h \in \mathscr{N}(\Omega) .
$$

Also, $u$ is in $J(\Omega)$ : With $\chi$ and $u_{2}$ as in the proof of Lemma 6.3, $\hat{f}:=P(L) u_{2}$ belongs to $\mathscr{S}\left(\mathbf{R}^{n}\right)$ and by Lemma 6.2 (i) there exists a unique solution $\hat{u} \in J\left(\mathbf{R}^{n}\right)$ of

Putting

$$
P(L) \hat{u}=\hat{f} .
$$

$$
v_{n^{\prime}}:=(1-\chi) u_{n^{\prime}}, \quad \hat{f}_{n^{\prime}}=P(L) v_{n}^{\prime}
$$

and continuing these functions by zero, we see from interior regularity results, that

$$
\lim _{n \rightarrow \infty}\left\|\left(1+|x|^{2}\right)^{p}\left(\hat{f}-\hat{f}_{n}\right)\right\|_{l, \mathbf{R}^{n}}=0 .
$$

Hence, by (6.5), $v_{n}$ tends to $\hat{u}$ in $H_{\mathrm{loc}}^{2 m}(\bar{\Omega})$. But then $u_{2}=\hat{u} \in J\left(\mathbf{R}^{n}\right)$; thus $u \in J(\Omega)$. It remains to lead the assumption

$$
\lim _{n \rightarrow \infty}\left\|u_{n}\right\|_{0, \Omega(S+1)}=\infty
$$


to a contradiction. For this we consider the normalized sequence

$$
w_{n}=\left\|u_{n}\right\|_{0, \Omega(S+1)^{-1} u_{n} .}
$$

As above we can conclude that a subsequence $w_{n}$, converges in $H_{\mathrm{loc}}^{2 m}(\bar{\Omega})$ to a solution $w \in J(\Omega)$ of $P(L) w=0, \gamma_{j} w=0$, which satisfies (6.16). By the choice of $\psi$ this implies $w=0$, which contradicts the normalization of $w_{n^{\prime}}$.

Let us now consider problem $(6.12 ; 6.13)$ with nonzero $g_{j}$. It is possible to construct a function $w \in H_{\mathrm{vox}}^{2 m}(\bar{\Omega})$ satisfying

$$
\begin{aligned}
& \gamma_{j} w=g_{j}, \quad j=0, \ldots, m-1, \\
& \text { supp } w \cap \operatorname{supp} \psi=\emptyset .
\end{aligned}
$$

Green's identity gives for $v \in \mathscr{N}^{*}(\Omega)$

$$
(P(L) w \mid v)_{0, \Omega}=\sum_{j=0}^{m-1}\left\langle g_{j} \mid T_{j} v\right\rangle_{0, \Gamma}
$$

Hence, if (6.14) is valid, we have

$$
(f-P(L) w \mid v)_{0, \Omega}=0 \text { for all } v \in \mathscr{N}^{*}(\Omega) .
$$

By the first part of the proof there is a solution $\tilde{u} \in J(\Omega)$ of $P(L) \tilde{u}=f-P(L) w$, $\gamma_{j} \tilde{u}=0$. Moreover, the function $\tilde{u}$ satisfies (6.16). Then the function

$$
u:=\tilde{u}+w
$$

solves $(6.12 ; 6.13)$ and, in addition, by (6.17), satisfies (6.16).

Since condition (6.14) is also necessary for the solvability, Theorem 6.4 is proved.

From the proof of Theorem 6.4 one easily deduces a pseudoinverse for problem $(6.12 ; 6.13)$

$$
\hat{\mathscr{P}}^{-1}: K(\Omega) \times \prod_{j=0}^{m-1} H^{2 m-j-1 / 2}(\Gamma) \rightarrow J(\Omega)
$$

which is continuous as a mapping from $H_{\mathrm{vox}}^{0}(\bar{\Omega}) \times \prod_{j=0}^{m-1} H^{2 m-j-1 / 2}(\Gamma)$ into $H_{\mathrm{loc}}^{2 m}(\bar{\Omega})$. For this we choose a nonnegative testfunction $\varphi \in \mathscr{D}(\Omega)$ such that the mapping $\mathscr{N}^{*}(\Omega) \rightarrow K(\Omega), v \rightarrow \varphi v$ is injective. Denoting by $v_{1}, \ldots, v_{d}$ a basis of $\mathscr{N}^{*}(\Omega)$ for which

the mapping

$$
\left(\varphi v_{i} \mid v_{j}\right)_{0, \Omega}=\delta_{i j}
$$

$$
\begin{aligned}
& Q: K(\Omega) \times \prod_{j=0}^{m-1} H^{2 m-j-1 / 2}(\Gamma) \rightarrow K(\Omega) \times \prod_{j=1}^{m-1} H^{2 m-j-1 / 2}(\Gamma), \\
& Q(f ; g)=\left(f-\sum_{i=1}^{d}\left(\left(f \mid v_{i}\right)_{0, \Omega}-\sum_{j=0}^{m-1}\left\langle g_{j} \mid T_{j} v_{i}\right\rangle_{0, \Gamma}\right) \varphi v_{i} ; g\right)
\end{aligned}
$$

is a continuous projection of $K(\Omega) \times \prod_{j=0}^{m-1} H^{2 m-j-1 / 2}(\Gamma)$ onto the range of the mapping $\mathscr{P}: J(\Omega) \rightarrow K(\Omega) \times \prod_{j=0}^{m-1} H^{2 m-j-1 / 2}(\Gamma)$, where

$$
\mathscr{P} u=\left(P(L) u ;\left.\gamma_{0} u\right|_{\Gamma}, \ldots,\left.\gamma_{m-1} u\right|_{\Gamma}\right)
$$


A pseudoinverse of $\mathscr{P}$ is given by assigning to any $\left(f ; g_{0}, \ldots, g_{m-1}\right)$ the unique solution $u \in J(\Omega)$ of

$$
P(L) u=\hat{f}, \quad \gamma_{j} u=g_{j}, \quad j=0, \ldots, m-1,
$$

with the property (6.16). Here $\left(\hat{f} ; g_{0}, \ldots, g_{m-1}\right)=Q\left(f, g_{0}, \ldots, g_{m-1}\right)$.

We have to show the continuity of $\hat{\mathscr{P}}^{-1}$. Since $Q$ is continuous, $\hat{\mathscr{P}}^{-1}$ is continuous if

$$
\|u\|_{2 m, \Omega(R)} \leqq c\left(\|P(L) u\|_{0, \Omega(S)}+\sum_{j=0}^{m-1}\left\|\gamma_{j} u\right\|_{2 m-j-1 / 2, \Gamma}\right)
$$

holds for any $u \in J(\Omega)$ with the properties (6.16-6.18). Estimate (6.19) has to be shown for any $R, S$, and the constant $c=c(R, S)$ may not depend on $u$. If (6.15) were wrong, there would exist numbers $R, S>0$ and a sequence $u_{k} \in J(\Omega)$ such that

$$
\begin{aligned}
& \left\|u_{k}\right\|_{2 m, \Omega(R)}=1, \\
& \lim _{k \rightarrow \infty}\left(\left\|P(L) u_{k}\right\|_{0, \Omega(S)}+\sum_{j=0}^{m-1}\left\|\gamma_{j} u_{k}\right\|_{2 m-j-1 / 2, \Gamma}\right)=0
\end{aligned}
$$

and that (6.15) are valid. Without loss of generality, we may suppose that $R \geqq S+1$. Then by Lemma 6.3 and Rellich's compactness theorem there exists a subsequence $u_{k^{\prime}}$, converging to some $u$ in $H_{\mathrm{loc}}^{2 m}(\bar{\Omega})$. As in the proof of Theorem 6.4, we can conclude, that $u$ belongs to $\mathscr{N}(\Omega)$ and hence vanishes by (6.16). This contradicts (6.20).

We have shown that for any exterior domain $\Omega$ with smooth boundary the Dirichlet operator, as defined by (6.18), is a weakly indexed operator with index 0 and admits a pseudoinverse $\hat{\mathscr{P}}^{-1}$ which is continuous as a mapping from $K(\Omega) \times \prod_{j=0}^{m-1} H^{2 m-j-1 / 2}(\Gamma)$ into $H_{\mathrm{loc}}^{2 m}(\bar{\Omega})$ and for which $Q=\mathscr{P} \hat{\mathcal{P}}^{-1}$ is a continuous projection of $K(\Omega)$ onto the range of $\mathscr{P}$. Since the adjoint Dirichlet problem is a problem of the same kind, this is true for $\mathscr{P}^{*}$ too. Therefore all assumptions (A1) - (A6) are valid in the case under discussion and one can conclude that Theorem 3.6 is applicable for exterior boundary value problems with respect to the operator $A=P(L)$.

Remark. To solve problem (2.15), one could proceed as in the proof of Theorem 6.4, using estimate (6.5) with $\gamma_{j}$ replaced by $B_{j}$. However, this would yield neither the index of the problem nor the finiteness of the codimension of the range.

\section{References}

[1] Agmon, S.: Lectures on elliptic boundary value problems. - D. Van Nostrand Company, Inc., Princeton, N. J. - Toronto, Ont.-New York-London, 1965.

[2] Agmon, S. and L. Hörmander: Asymptotic properties of solutions of differential equations with simple characteristics. - J. Analyse Math. 30, 1976, 1-38.

[3] Calderón, A. P.: Uniqueness in the Cauchy problem for partial differential equations. - Amer. J. Math. 80, 1958, 16-36. 
[4] Courant, R. and D. Hilbert: Methoden der mathematischen Physik I. - Verlag von Julius Springer, Berlin, 1931.

[5] Danilova, I. A.: Exterior boundary value problems for the Helmholtz equation. - Proc. Steklov Inst. Math. 103, 1970, 61-75.

[6] Edmunds, D. E. and W. D. Evans: Elliptic and degenerate-elliptic operators in unbounded domains. - Ann. Scuola Norm. Sup. Pisa (3) 27, 1973, 591-640.

[7] EĬdus, D. M.: The principle of limiting absorption. - Amer. Math. Soc. Transl. (2) 47, 1965, 157-191.

[8] El̆dus, D. M.: The principle of limit amplitude. - Russian Math. Surveys 24, 1969, 97-168.

[9] FINOŽENoK, G. N.: On boundary problems for equations of higher orders in infinite domains. Amer. Math. Soc. Transl. (2) 89, 1970, 251-273.

[10] Grušin, V. V.: On Sommerfeld-type conditions for a certain class of partial differential equations. - Amer. Math. Soc. Transl. (2) 51, 1966, 82-112.

[11] Hörmander, L.: Linear partial differential operators. - Springer Verlag, Berlin--HeidelbergNew York, 1969.

[12] Hörmander, L.: Lower bounds at infinity for solutions of differential equations with constant coefficients. - Israel J. Math. 16, 1973, 103-116.

[13] JÄGER, W.: Zur Theorie der Schwingungsgleichung mit variablen Koeffizienten in Außengebieten. - Math. Z. 102, 1967, 62-88.

[14] Jörgens, K.: Lineare Integraloperatoren. Mathematische Leitfäden. - B. G. Teubner, Stuttgart, 1970.

[15] KATO, T.: Growth properties of solutions of the reduced wave equation with a variable coefficient. - Comm. Pure Appl. Math. 12, 1959, 403-425.

[16] Kudruavcev, L. D.: The solution of the first boundary value problem for self-adjoint elliptic equations in the case of an unbounded region. - Math. USSR - Izv. 1, 1967, 11311151.

[17] LeIs, R.: Zur Monotonie der Eigenwerte selbstadjungierter elliptischer Differentialgleichungen. - Math. Z. 96, 1967, 26-32.

[18] LeIs, R.: Außenraumaufgaben zur Plattengleichung. - Arch. Rational Mech. Anal. 35, 1969, $226-233$.

[19] LeIs, R.: Zur Theorie der Plattengleichung. - Ber. Ges. Math. Datenverarb. Bonn 101, 1975.

[20] Levine, L. M.: A uniqueness theorem for the reduced wave equation. - Comm. Pure Appl. Math. 17, 1964, 147-176.

[21] Lions, J. L. and E. MAGENES: Non-homogeneous boundary value problems and applications I. - Springer Verlag, Berlin-Heidelberg-New York, 1972.

[22] Meyers, N. and J. Serrin: The exterior Dirichlet problem for second order elliptic partial differential equations. - J. Math. Mech. 9, 1960, 513-538.

[23] Neittanamäki, P.: Randwertaufgaben zur Plattengleichung. - Ann. Acad. Sci. Fenn. Ser. A I Math. Dissertationes 16, 1978, 1-71.

[24] Paneyah, B. P.: Existence and uniqueness of the solution of the $n$-metaharmonic equation on an unbounded case. - Vestnik Moscov. Univ. Ser. Mat. Meh. Astronom. Fiz. Him. 5, 1959, 123-135 (Russian).

[25] Pliś, A.: Non-uniqueness in Cauchy's problem for differential equations of elliptic type. M. Math. Mech. 9, 1960, 557-562.

[26] Pliś, A.: A smooth linear elliptic differential equation without any solution in a sphere. Comm. Pure Appl. Math. 14, 1961, 599-617.

[27] Polis, R.: Außenraumaufgaben in der Theorie der Plattengleichung. - Bonn. Math. Schr. No. 91, 1976.

[28] Protter, M. H.: Unique continuation for elliptic equations. - Trans. Amer. Math. Soc. 95, 1960, 81-91. 
[29] ReLlich, F.: Über das asymptotische Verhalten der Lösungen von $\Delta u+k^{2} u=0$ in unendlichen Gebieten. - J. Ber. Deutsch. Math. Verein. 53, 1943, 57-65.

[30] SAIto, Y.: The principle of limiting absorption for second-order differential equations with operator-valued coefficients. - Publ. Res. Inst. Math. Sci. 7, 1971/72, 581-619.

[31] Saranen, J.: A generalized plate equation with an exterior boundary value problem. - Ber. Univ. Jyväskylä Math. Inst. 17, 1977.

[32] Saranen, J.: On decomposition of solutions of some higher order elliptic equations. - Ann. Acad. Sci. Fenn. Ser. A I 4, 1978/79, 267-277.

[33] SARANen, J.: On electric and magnetic boundary problems for vector fields in nonhomogeneous anisotropic media. - J. Math. Anal. Appl. (to appear).

[34] Schulenberger, J. R. and C. H. Wilcox: A Rellich uniqueness theorem for steady-state wave propagation in inhomogeneous anisotropic media. - Arch. Rational Mech. Anal. 41, 1971, 18-45.

[35] VAǏNBERG, B. R.: Principles of radiation, limit absorption and limit amplitude in the general theory of partial differential equations. - Russian Math. Surveys 21, 115-163.

[36] VAĬNBERG, B. R.: On elliptic problems in unbounded domains. - Math. USSR-Sb. 4, 1968, $419-444$.

[37] Vekua, I. N.: New methods in solving elliptic boundary value problems. - John Wiley \& Sons, Inc., New York, 1967.

[38] Vogelsang, V.: Elliptische Differentialgleichungen mit variablen Koeffizienten in Gebieten mit unbeschränktem Rand. - Manuscripta Math. 14, 1975, 397-401.

[39] Vogelsang, V.: Das Ausstrahlungsproblem für elliptische Differentialgleichungen in Gebieten mit unbeschränktem Rand. - Math. Z. 144, 1975, 101-124.

[40] Wickel, W.: Außenraumaufgaben zur Plattengleichung mit Hilbertraummethoden. - Ber. Ges. Math. Datenverarb. Bonn 67, 1973.

[41] Wickel, W.: Zur Theorie der Dirichletschen Randwertaufgabe zum Operator $\Delta^{2}-k^{4}$ im Innen- und Außenraum mit der Integralgleichungsmethode. - Bonn. Math. Schr. No. $68,1973$.

[42] Wilcox, C. H.: Scattering theory for the d'Alembert equation in exterior domains. - Lecture Notes in Mathematics, 442, Springer-Verlag, Berlin-New York, 1975.

[43] Witsch, K. J.: Schiefe Außenraumaufgaben zu elliptischen Differentialgleichungen zweiter Ordnung. - Bonn. Math. Schr. No. 64, 1973.

[44] Witsch, K. J.: The exterior Dirichlet problem for a class of fourth order elliptic equations. J. Math. Anal. Appl. 49, 1975, 734-747.

[45] Witsch, K. J.: Radiation conditions and the exterior Dirichlet problem for a class of higher order elliptic equations. - J. Math. Anal. Appl. 54, 1976, 820-839.

J. Saranen
University of Oulu
Faculty of Technology
Department of Mathematics
Linnanmaa
SF-90570 Oulu 57
Finland

Received 21 January 1982
K. J. Witsch

University of Essen

FB 6 - Mathematics

Universitätsstraße 3

D 4300 Essen 1

German Federal Republic 